\title{
NONPARAMETRIC NEURAL NETWORK ESTIMATION OF LYAPUNOV EXPONENTS AND A DIRECT TEST FOR CHAOS ${ }^{*}$
}

\author{
by \\ Mototsugu Shintani \\ Vanderbilt University, Nashville, Tennessee \\ and \\ Oliver Linton \\ London School of Economics and Political Science
}

Contents:

Abstract

1. Introduction

2. Model

3. Theoretical results

4. Discussions

5. Simulation results

6. Application to financial data

7. Conclusion

Appendix

References

Tables $1-5$

Discussion Paper

No. EM/02/434

March 2002
The Suntory Centre

Suntory and Toyota International Centres for Economics and Related Disciplines London School of Economics and Political Science Houghton Street London WC2A 2AE Tel.: 020-7955 6698

* The authors thank Doug Nychka and Barb Bailey for providing their computer program. We also thank Xiaohong Chen, Rainer Dahlhaus, Yanqin Fan, Ron Gallant, Peter Phillips, Joon Park and Yoon Whang for comments, and the National Science Foundation for financial support. 


\begin{abstract}
This paper derives the asymptotic distribution of nonparametric neural network estimator of the Lyapunov exponent in a noisy system proposed by Nychka et al (1992) and others. Positivity of the Lyapunov exponent is an operational definition of chaos. We introduce a statistical framework for testing the chaotic hypothesis based on the estimated Lyapunov exponents and a consistent variance estimator. A simulation study to evaluate small sample performance is reported. We also apply our procedures to daily stock return datasets. In most cases we strongly reject the hypothesis of chaos; one mild exception is in some higher power transformed absolute returns, where we still find evidence against the hypothesis but it is somewhat weaker.
\end{abstract}

Keywords: Artificial neural networks; nonlinear dynamics; nonlinear time series; nonparametric regression; Sieve estimation.

JEL nos.: C14, C22.

(C) by the authors. All rights reserved. Short sections of text, not to exceed two paragraphs, may be quoted without special permission, provided that full credit, including (C) notice, is given to the source.

Contact address: Professor Oliver Linton, Department of Economics, London School of Economics and Political Science, Houghton Street, London WC2A 2AE, UK. Email: o.linton@lse.ac.uk/ 


\section{Introduction}

The largest Lyapunov exponent, which measures the average rate of divergence or convergence of two nearby trajectories, is a useful measure of the stability of a dynamical system. Indeed, the positivity of the Lyapunov exponent in a bounded dissipative nonlinear system is a widely used formal definition of chaos. ${ }^{1}$ To obtain the Lyapunov exponent from observed data, Eckmann and Ruelle (1985) and Eckmann et al. (1986) proposed a method based on nonparametric regression which is known as the Jacobian method. The Jacobian method is also applicable when stochastic noise is present in the system (Nychka et al., 1992). In recent work, the asymptotic distribution of the Jacobian method estimator using a kernel-type nonparametric regression in a noisy system was derived by Whang and Linton (1999). This asymptotic result can be used to test the null hypothesis of positive exponent as a formal test for chaos. ${ }^{2}$ In principle, any nonparametric regression estimator can be employed in the Jacobian method. Following the works by Nychka et al.(1992) and Gençay and Dechert (1992), the neural network became one of the most widely used nonparametric regression methods in the context of Lyapunov exponent estimation. ${ }^{3}$ However, despite the popularity of the neural networkbased Jacobian method, empirical researchers have been confined to report only the point estimates of the Lyapunov exponent as the distributional theory was not known. ${ }^{4}$

This paper first derives the asymptotic distribution of the Lyapunov exponent estimator based on neural networks. Based on the limiting distribution, consistent estimation of the variance of the Lyapunov exponent estimator is introduced. Then we propose a formal statistical framework for testing the hypothesis of the positivity of the Lyapunov exponent; in other words, we propose a direct test for chaos. ${ }^{5}$ The basic idea is to combine the result of Whang and Linton (1999) and the recent results on neural network asymptotics obtained by Chen and White (1999) and others. The conditions, in terms of the number of hidden units in neural nets as well as the block length, for asymptotic normality of the Lyapunov exponent estimator are derived for both one-dimensional and

\footnotetext{
${ }^{1}$ This definition is introduced by Eckmann and Ruelle (1985).

${ }^{2}$ Chaos can be defined in stochastic system using Lyapunov exponent. Such a generalization of the notion of chaos is sometimes referred to as noisy chaos as opposed to the deterministic chaos.

${ }^{3}$ See also McCaffrey (1991), Ellner et al. (1991) and McCaffrey et al. (1992) for the Jacobian method based on neural networks. Applications of this method in economics include: Dechert and Gençay's (1992) analysis of foreign exchange rates; studies on monetary aggregates by Serletis (1995) and Barnett et al. (1995); and the analysis of stock return series by Abhyankar, Copeland and Wong (1996).

${ }^{4}$ The bootstrap may be an alternative way to conduct a statistical test for the Lyapunov exponent. This line of research is pursued by Gençay (1996). However, the computing burden of bootstrapping neural nets seems to be the main problem in practice.

${ }^{5}$ The well-known BDS test proposed by Brock et al. (1996) should be viewed as a test for iid against general dependence which include chaos rather than a direct test for chaos.
} 
multi-dimensional cases. It is shown that the required growth rate of block length based on neural nets is slower than those based on kernel estimators.

Theoretically, neural nets are expected to perform better than other approximation methods especially with high-dimensional models since the approximation form is not sensitive to the increasing dimension. ${ }^{6}$ Practically, the reliability of the Jacobian method based on nonparametric neural network estimation is reaffirmed by a single-blind controlled competition conducted by Barnett et al. (1997). ${ }^{7}$ Using the data set used in this competition as well as using the artificially generated chaotic data in noisy system, the small sample properties of our proposed methods are examined. Finally, we apply our methods to stock return series. This is well-motivated since a certain type of economic model predicts chaos in the financial market.

The remainder of the paper is organized as follows: Definitions of the Lyapunov exponent and the neural network estimator are presented in Section 2. Section 3 derives asymptotic properties of estimated Lyapunov exponents based on neural nets and proposes test statistics. Some additional discussion is given in Section 4. Monte Carlo evidence is presented in Section 5. An empirical application is reported in Section 6. Some concluding remarks are made in Section 7. All proofs are given in the Appendix.

We will use following notation throughout the paper. When $|\cdot|$ is applied to a vector $a^{\prime}=$ $\left(a_{1}, \ldots, a_{d}\right) \in R^{d}$, it denotes a vector norm defined by $|a| \equiv \sum_{i=1}^{d}\left|a_{i}\right|$. Let $\mu=\left(\mu_{1}, \ldots, \mu_{d}\right)^{\prime}$ denote a $d$-vector of non-negative integer constants, we denote $x^{\mu}=\prod_{i=1}^{d} x_{i}^{\mu_{i}}$ for $x=\left(x_{1}, \ldots, x_{d}\right)^{\prime} \in \mathbb{R}^{d}$ and

$$
D^{\mu} g(x)=\frac{\partial^{|\mu|} g(x)}{\partial x_{1}^{\mu_{1}}, \ldots, \partial x_{d}^{\mu_{d}}},
$$

for any real function $g(x)$ on $\mathbb{R}^{d}$. When $\mu$ is a scalar constant, as is the case when $d=1$, we define $D^{\mu} g(x)$ to be the $\mu$-th order derivative of $g(\cdot)$ evaluated at $x$ with the convention that $D^{0} g(x)=g(x)$ and $D^{1} g(x)=D g(x)$. We use $\mathcal{B}_{d}^{m}$ to denote a weighted Sobolev space of all functions on $\mathbb{R}^{d}$ that have continuous and uniformly bounded (partial) derivative up to order $m$. For $g \in \mathcal{B}_{d}^{m}$, the norm is defined by

$$
\|g\|_{\mathcal{B}_{d}^{m}} \stackrel{\text { def. }}{=} \max _{0 \leq|\mu| \leq m_{x \in \mathbb{R}^{d}}} \sup _{x}\left|D^{\mu} g(x)\right|<\infty
$$

and the associated metric is defined with this norm. The symbols " $\Rightarrow$ " and " $\stackrel{p}{\rightarrow}$ are used to signify convergence in distribution and convergence in probability, respectively. All the limits in the paper are taken as the sample size $T \rightarrow \infty$.

\footnotetext{
${ }^{6}$ As stated in McCaffery et al. (1992) "Compared to the preceding functional approximations, the neural net form is not sensitive to increasing $d$ (p. 689)."

${ }^{7}$ Furthermore, the robustness of the neural nets to the choice of smoothing parameters (or number of hidden units) and lag length (or dimension) have been reported in simulations. See Gallant and White (1992) and Nychka et al. (1992).
} 


\section{Model}

\subsection{Lyapunov exponents for stochastic systems}

Let $\left\{X_{t}\right\}_{t=1}^{T}$ be a random scalar sequence generated by the following non-linear autoregressive model

$$
X_{t}=\theta_{0}\left(X_{t-1}, \ldots, X_{t-d}\right)+u_{t}
$$

where $\theta_{0}: \mathbb{R}^{d} \rightarrow \mathbb{R}$ is a non-linear dynamic map. We employ the following assumptions for the data $Z_{t}=\left(X_{t}, \ldots, X_{t-d+1}\right)^{\prime} \in \mathbb{R}^{d}$, error $u_{t}$, and the class of non-linear function $\theta_{0}$ in (1).

Assumption A1. (a) $\left\{Z_{t}\right\}_{t=1}^{T}$ is a strictly stationary and $\beta$-mixing sequence with mixing coefficient satisfying $\beta(j) \leq \beta_{0} j^{-\zeta}$ for some $\beta_{0}>0, \zeta>2$, where the $\beta$-mixing coefficient is given by

$$
\beta(j)=E \sup \left\{\left|P\left(B \mid \mathcal{F}_{-\infty}^{0}\right)-P(B)\right|: B \in \mathcal{F}_{j}^{\infty}\right\},
$$

where $\mathcal{F}_{s}^{t}$ is the $\sigma$-field generated by $\left(Z_{s}, \ldots, Z_{t}\right)$.

(b) The distribution of $Z_{t}$ is absolutely continuous with respect to Lebesgue measure with marginal density function $f$ with a compact support $\mathcal{Z}$ in $\mathbb{R}^{d}$. The initial condition $Z_{0}$ is a random variable generated from the same distribution.

Assumption A2. $\left\{u_{t}\right\}_{t=1}^{T}$ is a random sequence of either:

(i) i.i.d. with $E\left(u_{t}\right)=0$ and $E\left(u_{t}^{2}\right)=\sigma^{2}<\infty$, or

(ii) martingale difference with $E\left(u_{t} \mid \mathcal{F}_{-\infty}^{t-1}\right)=0$ and $E\left(u_{t}^{2} \mid \mathcal{F}_{-\infty}^{t-1}\right)=\sigma_{t}^{2} \in\left[\epsilon, \epsilon^{-1}\right]$ for some $\epsilon>0$.

Assumption A3. $\theta_{0}: \mathbb{R}^{d} \rightarrow \mathbb{R}$ is a target function in the parameter space having finite third absolute moments of the Fourier magnitude distributions, namely,

$$
\Theta=\left\{\theta: \theta(z)=\int \exp \left(i a^{\prime} z\right) d \mu_{\theta}(a), \quad\left\|\mu_{\theta}\right\|_{3} \equiv \int l(a)^{3} d\left|\mu_{\theta}\right|(a) \leq C<\infty\right\},
$$

where $\mu_{\theta}$ is a complex-valued measure on $\mathbb{R}^{d},\left|\mu_{\theta}\right|$ denotes total variation of $\mu_{\theta}, l(a)=\max \left[\left(a^{\prime} a\right)^{1 / 2}, 1\right]$ and $a^{\prime}=\left(a_{1}, \ldots, a_{d}\right) \in \mathbb{R}^{d}$.

Assumption A3 is a slightly modified version of the smoothness condition first introduced by Barron (1993) in the neural network literature. For the purpose of investigating approximation properties of neural nets with respect to derivatives up to order $m$, the modified norm with the 
scaling factor $l(a)^{m+1}$ is introduced by Hornik et al. (1994). It should be noted that such a modified condition is stronger than Barron's original condition and requires the Sobolev norm of order $m+1$ to be bounded. ${ }^{8}$ In other words, A3 implies that $\theta \in \mathcal{B}_{d}^{3}$ or boundedness of the third derivative. This fact will be used later as a bridge between convergence in $L_{2}$ norm and Sobolev norm to obtain uniform convergence rate of derivative estimation from neural networks (see Lemma 1).

The model (1) can be expressed in terms of a map with $U_{t}=\left(u_{t}, 0, \ldots, 0\right)^{\prime}$ and the map function $F: \mathbb{R}^{d} \rightarrow \mathbb{R}^{d}$ such that

$$
Z_{t}=F\left(Z_{t-1}\right)+U_{t}
$$

Let $J_{t}$ be the Jacobian of the map $F$ in (2) evaluated at $Z_{t}$. Specifically, we define

$$
J_{t}=\left[\begin{array}{ccccc}
\Delta \theta_{1 t} & \Delta \theta_{2 t} & \cdots & \Delta \theta_{d-1, t} & \Delta \theta_{d t} \\
1 & 0 & \cdots & 0 & 0 \\
0 & 1 & \cdots & 0 & 0 \\
\vdots & \vdots & \ddots & \vdots & \vdots \\
0 & 0 & \cdots & 1 & 0
\end{array}\right]
$$

for $t=0,1, \ldots, T-1$, where $\Delta \theta_{j t}=D^{e_{j}} \theta_{0}\left(Z_{t}\right)$ for $j=1, \ldots, d$ in which $e_{j}=(0, \ldots, 1, \ldots, 0)^{\prime} \in \mathbb{R}^{d}$ denotes the $j$-th elementary vector. We next assume the existence of the Lyapunov exponent of (1).

Assumption A4. The system (1) has a finite Lyapunov exponent defined by

$$
\lambda \equiv \lim _{M \rightarrow \infty} \frac{1}{2 M} \ln \nu_{1}\left(\mathbf{T}_{M}^{\prime} \mathbf{T}_{M}\right), \quad \mathbf{T}_{M}=\prod_{t=1}^{M} J_{M-t}=J_{M-1} \cdot J_{M-2} \cdots J_{0},
$$

where $\nu_{i}(A)$ is $i$-th largest eigenvalue of a matrix $A$.

Necessary conditions for Assumption A4 have been discussed in the literature [For example, see Nychka et al., 1992, p.406]. It is known that, if $\max \left\{\ln \nu_{1}\left(J_{t}^{\prime} J_{t}\right), 0\right\}$ has a finite first moment with respect to the distribution given in Assumption A1, then the limit in (4) almost surely exists and will be a constant, irrespective of the initial condition. When $\sigma_{t}^{2}=0$, the system (1) reduces to a deterministic system and the interpretation of $\lambda>0$ is identical to the definition of deterministic chaos. For moderate $\sigma_{t}^{2}$, the stochastic system generated by (1) can also have sensitive dependence to initial conditions, and noisy chaos with $\lambda>0$ can be also defined (see Nychka et al., 1992). For example, a stationary linear autoregressive process has $\lambda<0$, while the unit root and the explosive autoregressive process imply $\lambda \geq 0$. One interesting question here is whether the Lyapunov exponent is continuous in the amount of noise for small amounts of noise. Specifically, let $\lambda_{\sigma}$ denote the

\footnotetext{
${ }^{8}$ Barron's original condition requires only the Sobolev norm of order 1 to be bounded.
} 
Lyapunov exponent for a noisy system with error variance $\sigma^{2}$ and let $\lambda_{0}$ be the Lyapunov exponent for the deterministic skeleton with $\sigma^{2}=0$. We suspect that $\lim _{\sigma \rightarrow 0} \lambda_{\sigma}=\lambda_{0}$. This is certainly the case for a large class of processes including the linear autoregressive processes, but we do not have a proof that works under general conditions. Under further continuity properties, our distributional theory given below can also be extended to 'small sigma' asymptotics, i.e., to work under the condition that $\sigma \rightarrow 0$.

For the multidimensional case with $d \geq 2$, we can also define the $i$-th largest Lyapunov exponents $\left(\lambda_{i}\right)$ for $2 \leq i \leq d$, by replacing $\nu_{1}(\cdot)$ in (4) by $\nu_{i}(\cdot)$ for $2 \leq i \leq d$. These other exponents also contain some important information related to the stability of the system, including the directions of divergence and contraction of trajectories (see Nychka et al., 1992) and the types of non-chaotic attractors (see Dechert and Gençay, 1992). In the following subsection, we introduce the single hidden layer networks to obtain the nonparametric estimates of (1) and (4).

\subsection{Nonparametric neural network estimation}

Let $\widehat{\theta}(\cdot)$ be the estimate of $\theta_{0}(\cdot)$ in $(1)$ based on neural networks. In this paper, we consider only feed-forward single hidden layer networks with a single output, which is the most frequently used model in the statistical analysis on neural nets. Following Chen and Shen (1998) and Chen and White (1999), we view this neural network estimator as a special case of Grenander's (1981) sieve extremum estimator in our analysis. In other words, it can be viewed as a problem of maximizing an empirical criterion, $L_{T}(\theta)$, over the neural network sieve, $\Theta_{T}$, which is a sequence of approximating parameter spaces that is dense in the infinite dimensional parameter space, $\Theta$, as $T \rightarrow \infty$. The formal definition of the estimator is given as follows.

Assumption B1. (a) The neural network estimator $\widehat{\theta}_{T}$ is an extremum sieve estimator that satisfies

$$
L_{T}\left(\widehat{\theta}_{T}\right) \geq \sup _{\theta \in \Theta_{T}} L_{T}(\theta)-O\left(\varepsilon_{T}^{2}\right)
$$

with $\varepsilon_{T} \rightarrow 0$ as $T \rightarrow \infty$, where $L_{T}(\theta)$ is a least square criterion

$$
L_{T}(\theta)=\frac{1}{T} \sum_{t=1}^{T} l\left(\theta, X_{t}, Z_{t-1}\right)=-\frac{1}{T} \sum_{t=1}^{T} \frac{1}{2}\left(X_{t}-\theta\left(Z_{t-1}\right)\right)^{2} .
$$

(b) The neural network sieve $\theta_{T}: \mathbb{R}^{d} \rightarrow \mathbb{R}$ is an approximation function in the parameter space $\Theta_{T}$ satisfying

$$
\theta_{T}(z)=\beta_{0}+\sum_{j=1}^{2^{k} r(T)} \beta_{j} l\left(a_{j}\right)^{-3} \psi\left(a_{j}^{\prime} z+b_{j}\right)
$$


with

$$
\max _{1 \leq j \leq 2^{k} r(T)}\left|a_{j}\right| \leq C_{T}, \quad \sum_{j=0}^{2^{k} r(T)}\left|\beta_{j}\right| \leq B_{T}
$$

where $\psi$ is an activation function, $a_{j} \in \mathbb{R}^{d}, b_{j}, \beta_{j} \in \mathbb{R}$ are parameters, and $k$ is the number related to the choice of activation function defined below.

In B1(a), we consider the approximate maximization problem where exact maximization is included as a special case when $\varepsilon_{T}=0$. Similar to the case shown in Chen and Shen (1998), our asymptotics in the next section are valid as long as $\varepsilon_{T}$ converges to zero faster than the theoretical rate of convergence of the estimator. The neural network sieve in B2(b) is again slightly different from the usual definition since each hidden unit is scaled by the factor $l\left(a_{j}\right)^{-3}$ for the same reason as A3. Also note that $2^{k} r(T)$ is the number of hidden units instead of $r(T)$ in our notation.

Typically, $\psi$ is a sigmoid function defined by a bounded measurable function on $\mathbb{R}$ with $\psi(u) \rightarrow 1$ as $u \rightarrow \infty$, and $\psi(u) \rightarrow 0$ as $u \rightarrow-\infty$. However, we do not need to restrict our attention to sigmoid functions in order to obtain the powerful approximation properties of the neural networks. For this reason, we employ the condition of Hornik et al. (1994) and allow a more general class of functions for our activation function.

Assumption B2 (Hornik et al., 1994). The activation function $\psi$ is a possibly nonsigmoid function satisfying $\psi \in \mathcal{B}_{1}^{2}$ and is $k$-finite for some $k \geq 2$, namely,

$$
0<\int_{\mathbb{R}}\left|D^{k} \psi(u)\right| d u<\infty .
$$

We can replace the condition above by the stronger condition that all derivatives $D^{k} \psi, 0<k<2$, are absolutely integrable with respect to one-dimensional Lebesgue measure, or, $\int_{\mathbb{R}}\left|D^{k} \psi(u)\right| d u<\infty$ for all $k$ such that $0<k<2$.

We now define our estimator of $\lambda$ based on the neural network estimator $\widehat{\theta}$. The basic idea of the Jacobian method is to substitute $\widehat{\theta}$ in the Jacobian formula (3) and obtain $\widehat{J}_{t}$. Following the convention of neural network estimation of Lyapunov exponent, we distinguish between the "sample size" $T$ used for estimating Jacobian $\widehat{J}_{t}$ and the "block length" $M$ which is the number of evaluation points used for estimating Lyapunov exponent. Since the number of evaluation points should always be less than or equal to $T, M$ can be also understood as a subsample. The neural network estimator of Lyapunov exponent can be defined as

$$
\widehat{\lambda}_{M}=\frac{1}{2 M} \ln \nu_{1}\left(\widehat{\mathbf{T}}_{M}^{\prime} \widehat{\mathbf{T}}_{M}\right), \quad \widehat{\mathbf{T}}_{M}=\prod_{t=1}^{M} \widehat{J}_{M-t}=\widehat{J}_{M-1} \cdot \widehat{J}_{M-2} \cdots \widehat{J}_{0},
$$


where

$$
\widehat{J}_{t}=\left[\begin{array}{ccccc}
\Delta \widehat{\theta}_{1 t} & \Delta \widehat{\theta}_{2 t} & \cdots & \Delta \widehat{\theta}_{d-1, t} & \Delta \widehat{\theta}_{d t} \\
1 & 0 & \cdots & 0 & 0 \\
0 & 1 & \cdots & 0 & 0 \\
\vdots & \vdots & \ddots & \vdots & \vdots \\
0 & 0 & \cdots & 1 & 0
\end{array}\right]
$$

where $\Delta \widehat{\theta}_{j t}=D^{e_{j}} \widehat{\theta}\left(Z_{t}\right)$ for $t=0,1, \ldots, M-1$. For notational convenience we have just taken the first $M$ observations. However, in practice, there are several alternative choices of subsample, a matter that will be discussed in subsection 4.2. The estimator for the other Lyapunov exponents, $\widehat{\lambda}_{i M}$, can be defined similarly by replacing $\nu_{1}(\cdot)$ by $\nu_{i}(\cdot)$ for $2 \leq i \leq d$.

\section{Theoretical results}

\subsection{Uniform convergence rate for derivative estimator}

We first provide the uniform convergence rate for the derivative estimator. Let us denote $\Delta \widehat{\theta}\left(Z_{t}\right)=$ $\left(\Delta \widehat{\theta}_{1, t}, \Delta \widehat{\theta}_{2, t}, \ldots, \Delta \widehat{\theta}_{d, t}\right)^{\prime}$ and $\Delta \theta_{0}\left(Z_{t}\right)=\left(\Delta \theta_{01, t}, \Delta \theta_{02, t}, \ldots, \Delta \theta_{0 d, t}\right)^{\prime}$.

Lemma 1. Suppose that assumptions $A 1$ to $A 4$ and B1 to B2 hold, $B_{T} \geq$ const. $\times\left\|\mu_{\theta}\right\|_{3}$, $C_{T}=$ const. and $r(T)$ satisfies $r^{2} \log r=O(T)$. Then

$$
\sup _{z \in \mathcal{Z}}\left|\Delta \widehat{\theta}(z)-\Delta \theta_{0}(z)\right|=O_{p}\left([T / \log T]^{-1 / 4}\right) .
$$

In order to obtain the improved rate for the derivative estimator, we introduce a Hölder condition on the activation function. ${ }^{9}$

Assumption B3 (Chen and White, 1999). For any $\left(a^{\prime}, b\right),\left(a_{1}^{\prime}, b_{1}\right) \in \mathbb{R}^{d} \times \mathbb{R}$, there exists an $\alpha \in(0,1]$ associated with $\psi \in \mathcal{B}_{1}^{3}$ such that for all $z$ in the compact support $S$,

$$
\left\|\psi_{a, b}-\psi_{a_{1}, b_{1}}\right\|_{\mathcal{B}_{1}^{3}} \leq \text { const. } \times\left[\left(\left(a-a_{1}\right)^{\prime}\left(a-a_{1}\right)\right)^{1 / 2}+\left|b-b_{1}\right|\right]^{\alpha},
$$

where $\psi_{a, b}(z)$ is the rescaled activation function defined by $\psi_{a, b}(z)=l(a)^{-3} \psi\left(a^{\prime} z+b\right)$.

Lemma 2. Suppose that assumptions $A 1$ to $A 4$ and $B 1$ to B3 hold, $B_{T} \geq$ const. $\times\left\|\mu_{\theta}\right\|_{3}, C_{T}=$ const. and $r(T)$ satisfies $r^{2\left(1+\alpha / d^{*}\right)} \log r=O(T)$, where $d^{*}=d$ if $\psi$ is homogeneous $(\psi(c z)=c \psi(z))$,

\footnotetext{
${ }^{9}$ See Makovoz (1996) and Chen and White (1999) for the relation between this condition and the source of improvement in the rate of approximation.
} 
and $d^{*}=d+1$ otherwise. Then

$$
\sup _{z \in \mathcal{Z}}\left|\Delta \widehat{\theta}(z)-\Delta \theta_{0}(z)\right|=o_{p}\left(T^{-1 / 4}\right) .
$$

\subsection{Lyapunov exponent when $d=1$}

In this subsection, the asymptotic behavior of $\widehat{\lambda}$ for the scalar case $(d=1)$ is presented mainly for the purpose of illustration. The general results for the multidimensional case $(d \geq 2)$ will be obtained in the next subsection. When $d=1$, since $Z_{t}=X_{t}, \mathcal{Z}=\chi$, and $J_{t}=D \theta_{0}\left(X_{t}\right)$, the Lyapunov exponent estimator in (5) simplifies to

$$
\widehat{\lambda}_{M}=\frac{1}{2 M} \sum_{t=1}^{M} \ln \left[D \widehat{\theta}\left(X_{t-1}\right)^{2}\right] .
$$

To investigate the asymptotic property of the estimator, it is convenient to introduce the notion of the local Lyapunov exponent defined by

$$
\lambda_{M}=\frac{1}{2 M} \sum_{t=1}^{M} \ln \left[D \theta\left(X_{t-1}\right)^{2}\right] .
$$

Unlike the "global" Lyapunov exponent $\lambda$, the local Lyapunov exponent measures the short-term rate of divergence. It should also be noted that $\lambda_{M}$ is a random variable in general. By the definition in assumption A4, $\lambda$ can be seen as a limit of $\lambda_{M}$ with $M \rightarrow \infty$. Using $\lambda_{M}$, the total estimation error, $\widehat{\lambda}_{M}-\lambda$, with normalizer $\sqrt{M}$ can be decomposed as

$$
\sqrt{M}\left(\widehat{\lambda}_{M}-\lambda\right)=\sqrt{M}\left(\widehat{\lambda}_{M}-\lambda_{M}\right)+\sqrt{M}\left(\lambda_{M}-\lambda\right) .
$$

The second term represents the asymptotic behavior of the local Lyapunov exponent which is common to all Jacobian methods irrespective of the choice of the nonparametric estimator. The $\sqrt{M}$ rate of convergence and CLT for this term were derived by McCaffrey et al. (1992) and Bailey (1996), respectively. The first term can be understood as the estimation error for the local Lyapunov exponent. In contrast to the second term, the asymptotic behavior of the first term depends on the estimation method. Whang and Linton (1999) employed kernel regression methods and showed that the asymptotic of (7) is dominated by the second term under some conditions. Similarly, we investigate the condition on the neural network estimator for the first term to have negligible effect on the asymptotic behavior of (7). To obtain asymptotic normality, we employ the following two assumptions.

Assumption A5*. For some $\phi \geq 0$,

$$
\max _{1 \leq t \leq M}\left(\left|D \theta_{0}\left(X_{t-1}\right)\right|^{-1}\right)=\left(\min _{1 \leq t \leq M}\left|D \theta_{0}\left(X_{t-1}\right)\right|\right)^{-1}=O_{p}\left(M^{\phi}\right) .
$$


Assumption A6*.

$$
\Phi \equiv \lim _{M \rightarrow \infty} \operatorname{var}\left[\frac{1}{\sqrt{M}} \sum_{t=1}^{M} \eta_{t}\right]
$$

is positive and finite, where

$$
\eta_{t}=\ln \left|D \theta_{0}\left(X_{t-1}\right)\right|-\lambda
$$

Assumption $\mathrm{A} 5^{*}$ is a condition on the properties of the data around zero derivatives first employed by Whang and Linton (1999). As explained in Whang and Linton (p. 9), it is closely related to extreme value theory for stochastic processes. The condition is weak and is expected to hold for many chaotic processes including the well-known logistic map. Assumption A6* provides the variance of the second term in (7), namely, the variance for the local Lyapunov exponent. The following points should be noted with respect to this assumption. Since $\eta_{t}$ is a weakly dependent process, if we take an equally spaced subsample, it is expected to be an asymptotically independent sequence. Also, this condition implies that we are focusing our attention on the nonlinear case, since the variance degenerates in the linear case. Using these assumptions, we obtain the following result.

Theorem 1. Suppose that assumptions in Lemma 1, A5* and $A 6^{*}$ hold. If $M=O\left([T / \log T]^{1 /(2+4 \phi)}\right)$, then

$$
\sqrt{M}\left(\widehat{\lambda}_{M}-\lambda\right) \Rightarrow N(0, \Phi)
$$

Remark. The result shows that the simple asymptotic formula can be derived with an appropriate choice of block length. For example, when $\phi=0, M=O\left([T / \log T]^{1 / 2}\right)$ is sufficient for the first term in (7) to have negligible effect in the asymptotic distribution. It is also interesting to compare the required conditions on block length with those of kernel based method derived in Whang and Linton (1999). First, unlike our result, their rate depends on the trimming parameter because of the boundary problem of kernel estimation. ${ }^{10}$ Second, the difference in the convergence rate of the derivative estimator results in a slower growth rate of the block length in the neural network procedure than those in the kernel based method by the logarithmic term in the denominator.

\subsection{Lyapunov exponents when $d \geq 2$}

\footnotetext{
${ }^{10}$ It should also be noted that Whang and Linton (1999) allow their rate of bandwidth to be random, but we restrict our attention to the deterministic number of hidden units.
} 
We now discuss the extension of the result in the previous subsection to the case in which $d$ may be greater than one. For the purpose of deriving the asymptotic properties, we will introduce multidimensional version of assumptions $\mathrm{A} 5^{*}$ and $\mathrm{A} 6^{*}$ for the one-dimensional case.

Assumption A5. For $1 \leq i \leq d$ and some $\phi \geq 0$,

$$
\max _{1 \leq t \leq M}\left|F_{i t}\left(J_{M-1}, \ldots, J_{0}\right)\right|=O_{p}\left(M^{\phi}\right)
$$

where

$$
F_{i t}\left(J_{M-1}, \ldots, J_{0}\right)=\frac{\partial \ln \nu_{i}\left(\mathbf{T}_{M}^{\prime} \mathbf{T}_{M}\right)}{\partial \Delta \theta\left(Z_{t}\right)} \text { and } \Delta \theta\left(Z_{t}\right)=\left(\Delta \theta_{1, t}, \Delta \theta_{2, t}, \ldots, \Delta \theta_{d, t}\right)^{\prime}
$$

Assumption A6. For $1 \leq i \leq d$,

$$
\Phi_{i} \equiv \lim _{M \rightarrow \infty} \operatorname{var}\left[\frac{1}{\sqrt{M}} \sum_{t=1}^{M} \eta_{i t}\right]
$$

is positive and finite, where

$$
\eta_{i t}=\xi_{i t}-\lambda_{i} \text { with } \xi_{i t}=\frac{1}{2} \ln \left(\frac{\nu_{i}\left(\mathbf{T}_{t}^{\prime} \mathbf{T}_{t}\right)}{\nu_{i}\left(\mathbf{T}_{t-1}^{\prime} \mathbf{T}_{t-1}\right)}\right) \text { for } t \geq 2 \text { and } \xi_{i 1}=\frac{1}{2} \ln \nu_{i}\left(\mathbf{T}_{1}^{\prime} \mathbf{T}_{1}\right)
$$

By using arguments similar to those of the previous subsection, we have the main theoretical result of the paper.

Theorem 2. Suppose that assumptions in Lemma 1, A5 and A6 hold. If $M=O\left([T / \log T]^{1 /(2+4 \phi)}\right)$, then for $1 \leq i \leq d$,

$$
\sqrt{M}\left(\widehat{\lambda}_{i M}-\lambda_{i}\right) \Rightarrow N\left(0, \Phi_{i}\right)
$$

Remark. The result shows that a simple asymptotic formula can be used for all Lyapunov exponents in the multidimensional case, with the growth rate of the block length identical to the rate which applies in the one-dimensional case. It should also be noted that both one-dimensional and multidimensional results are obtained using the same smoothness condition given in A3. This advantage of the neural network approach comes from the powerful approximation properties of neural networks given in Lemma 1. In general, other nonparametric approaches require stronger conditions on the smoothness (or differentiability) of the function when the dimension increases (for example, see the requirement for the kernel regression based method in Whang and Linton, 1999). 


\subsection{Test statistics}

In this subsection, feasible test statistics are introduced and a one-sided test is proposed for the purpose of testing chaotic behavior of time series. First, we construct the test statistics based on the asymptotic results on Lyapunov exponent estimates obtained in the previous subsections. To simplify the notation, we omit the subscript 1 for the largest Lyapunov exponent in the multidimensional case. Suppose $\widehat{\Phi}$ is a consistent estimator of $\Phi$ in Theorem 2. Our primary interest is to test the null hypothesis $H_{0}: \lambda \geq 0(\lambda \leq 0)$ against the alternative of $H_{1}: \lambda<0(\lambda>0)$. Our test statistic is

$$
\widehat{t}=\frac{\widehat{\lambda}_{M}}{\sqrt{\widehat{\Phi} / M}}
$$

We reject the null hypothesis if $\widehat{t} \leq-z_{\alpha}\left(\widehat{t} \geq z_{\alpha}\right)$ where $z_{\alpha}$ is the critical value that satisfies $\operatorname{Pr}\left[Z \geq z_{\alpha}\right]=\alpha$ with $Z$ being a standard normal random variable.

Next, we consider consistent estimation of $\Phi$. Since $\eta_{t}$ 's are serially dependent and not identically distributed, we need to employ a heteroskedasticity and autocorrelation consistent (HAC) covariance matrix estimator (see, e.g. Andrews, 1991) for $\Phi$. For the one-dimensional case, the covariance estimator $\widehat{\Phi}$ is defined as follows:

$$
\widehat{\Phi}=\sum_{j=-M+1}^{M-1} w\left(j / S_{M}\right) \widehat{\gamma}(j) \text { and } \widehat{\gamma}(j)=\frac{1}{M} \sum_{t=|j|+1}^{M} \widehat{\eta}_{t} \widehat{\eta}_{t-|j|}
$$

where $\widehat{\eta}_{t}=\left\{\ln \left|D \widehat{\theta}\left(X_{t-1}\right)\right|-\widehat{\lambda}_{M}\right\}$ and where $w(\cdot)$ and $S_{M}$ denote a kernel function and a lag truncation parameter, respectively. For the multidimensional case, the test statistic $\widehat{t}_{i}=\widehat{\lambda}_{i M} / \sqrt{\widehat{\Phi}_{i} / M}$ with the covariance estimators $\widehat{\Phi}_{i}$ can be similarly constructed by replacing $\widehat{\eta}_{t}$ by

$$
\widehat{\eta}_{i t}=\widehat{\xi}_{i t}-\widehat{\lambda}_{i M} \text { with } \widehat{\xi}_{i t}=\frac{1}{2} \ln \left(\frac{\nu_{i}\left(\widehat{\mathbf{T}}_{t}^{\prime} \widehat{\mathbf{T}}_{t}\right)}{\nu_{i}\left(\widehat{\mathbf{T}}_{t-1}^{\prime} \widehat{\mathbf{T}}_{t-1}\right)}\right) \text { for } t \geq 2 \text { and } \widehat{\xi}_{i 1}=\frac{1}{2} \ln \nu_{i}\left(\widehat{\mathbf{T}}_{1}^{\prime} \widehat{\mathbf{T}}_{1}\right) \text {. }
$$

For the covariance matrix estimation, we employ the following class of kernel functions similar to that given in Andrews (1991).

Assumption $\mathbf{C 1}$ (HAC estimation). $w: \mathbb{R} \rightarrow[-1,1]$ is a piecewise continuous function, continuous and taking the value 1 at zero, symmetric around zero, and has a finite second moment. In other words, the class of kernel is

$$
\begin{aligned}
& \mathcal{W}=\left\{w(\cdot): w(0)=1, w(-x)=w(x) \forall x \in \mathbb{R}, \int_{-\infty}^{\infty} w^{2}(x) d x<\infty,\right. \\
& w(\cdot) \text { is continuous at } 0 \text { and at all but a finite number of points }\} \text {. }
\end{aligned}
$$


Corollary 1. Suppose that assumptions in Theorem 2 and C1 are satisfied. Also suppose, $S_{M}$ satisfies $S_{M} \rightarrow \infty$ and $S_{M}=o\left(M^{1 / 2}\right)$. Then for $1 \leq i \leq d, \widehat{\Phi}_{i} \stackrel{p}{\rightarrow} \Phi_{i}$.

Remark. This result shows that the HAC estimation with given growth rate of bandwidth can be used to construct the standard error for Lyapunov exponents. Since the infeasible statistic $\widetilde{t}_{i}=\left(\widehat{\lambda}_{i M}-\lambda_{i}\right) / \sqrt{\widehat{\Phi}_{i} / M} \Rightarrow N(0,1), \widehat{t}_{i}=\widetilde{t}_{i}+\lambda_{i} / \sqrt{\widehat{\Phi}_{i} / M}$ diverges to $-\infty(\infty)$ for any $\lambda_{i}$ under $H_{1}$ : $\lambda_{i}<0\left(\lambda_{i}>0\right)$. Therefore the test is consistent under reasonable conditions.

\section{Discussions}

\subsection{Optimal block length and optimal subsampling scheme}

It should be noted that the asymptotic results in the previous section required that the number of products of the Jacobian in the Lyapunov exponent estimate $(M)$ to be less than the sample size of data used in Jacobian estimation $(T)$. Therefore, the choice of block length $M$ is an important issue in practice. McCaffrey et al. (1992) discussed the optimal choice of block length by decomposing the local Lyapunov exponent asymptotics [the second term in (7)] into a bias term and a variance term. Furthermore, they suggested that averaging the Lyapunov exponent estimators from the nonoverlapping $T / M$ blocks might reduce the overall bias (see also Ellner et al., 1991, and Nychka et al., 1992). However, it should be noted that such an estimate in the one-dimensional case is identical to the estimate based on a full sample $(M=T)$.

Whang and Linton (1999) pointed out that the asymptotics for the Lyapunov exponent estimate can be derived not only from the blocking method but also from any other subsampling method. This fact also raised a question of the optimal choice of subsampling scheme for a given number of $M$. Suppose the optimal choice is made on the grounds that it minimizes the variance $\Phi_{i}$ in $\mathrm{A} 6$ (or A $6^{*}$ ). Then the comparison between the blocking scheme and the equally spaced subsampling scheme can be understood from the following simple example.

Suppose we have three observations of the time series data $\left(y_{1}, y_{2}, y_{3}\right)$ generated from the autoregressive $(\mathrm{AR})$ process of order one. If we want to estimate the mean of the process using two observations out of three, we only have two alternatives; using the adjacent sample $\left[\left(y_{1}, y_{2}\right)\right.$ or $\left.\left(y_{2}, y_{3}\right)\right]$ or use the skipped sample $\left[\left(y_{1}, y_{3}\right)\right]$. The variance of such an estimate depends on the AR parameter. A simple calculation implies that the first scheme is more efficient when the parameter is negative and the second scheme is more efficient when the parameter is positive. Similarly, when the data are generated by the moving average (MA) process of order one, the first scheme is better when the MA parameter is positive and the second scheme is better when the parameter is negative. 
This simple example shows that the optimal subsample for the Lyapunov exponent estimation depends on the data generating process. Therefore, we may use either the blocking scheme or equally spaced subsample scheme as a choice of subsample. For this reason, in this paper, we report the results based on equally spaced subsamples in addition to the results based on the commonly used blocking method in the simulation and empirical analysis.

\subsection{Full sample estimation}

As discussed by Ellner et al. (1991), it has been questioned whether the requirement of block length $(M)$ less than full sample $(T)$ is necessary in the theoretical analysis of asymptotic behavior of the neural network approach. When the Jacobians from the whole sample points are used for Lyapunov exponent calculation $(M=T)$, the first term in (7) now enters the asymptotic behavior of the overall estimation error. Therefore, we can expect the full sample estimator to have a different asymptotic distribution from the one based on subsamples. Whang and Linton (1999) showed that the asymptotic distribution for a full sample estimator based on kernel regression can be derived if one employs some strong assumptions on the functional form. The purpose of this subsection is to illustrate that it is also possible in the neural network approach to derive the asymptotic results if the similar assumptions are employed. To simplify the argument, we only consider the one-dimensional case.

Corollary 2. Suppose that assumptions in Lemma 2, A5* with $\phi=0$ hold, $\eta_{t}$ in A6* is replaced by $\eta_{t}=v\left(X_{t-1}\right) u_{t}+\ln \left|D \theta_{0}\left(X_{t-1}\right)\right|-\lambda$, where

$$
v(x)=\frac{D^{2} \theta_{0}(x)}{\left\{D \theta_{0}(x)\right\}^{2}}-\frac{D f(x)}{\left\{D \theta_{0}(x)\right\} f(x)} .
$$

Further assume that $f(x) / D \theta_{0}(x)=0$ at the boundary points $\underline{x}$ and $\bar{x}$. Then we have the asymptotic normality result in Theorem 1 with $M=T$.

Remark. To derive this result, stronger conditions for both activation function and target function are employed. Among all additional conditions, $\phi=0$ is the most difficult requirement since it "is not satisfied by any univariate chaotic process that we are aware of (Whang and Linton, 1999, p.8)." The consistent estimator of $\Phi$ can be constructed by using the sample analogue of $\eta_{t}$, which requires a second derivative estimation of target function as well as density and density derivative estimation. 


\subsection{Upper bound estimation}

The definition of $\xi_{i t}$ in Theorem 2 does not have a simple form as $\xi_{t}$ in Theorem $1 \operatorname{since} \ln \nu_{i}\left(\mathbf{T}_{M}^{\prime} \mathbf{T}_{M}\right) \neq$ $\sum_{t=1}^{M} \ln \nu_{i}\left(J_{M-t}^{\prime} J_{M-t}\right)$ for the multivariate case. However for the largest Lyapunov exponent $(i=1)$, we have the following relation between the two quantities:

$$
\sum_{t=1}^{M} \ln \nu_{1}\left(J_{M-t}^{\prime} J_{M-t}\right)=\ln \prod_{t=1}^{M} \nu_{1}\left(J_{M-t}^{\prime} J_{M-t}\right) \geq \ln \nu_{1}\left(\left(\Pi_{i=1}^{M} J_{M-t}\right)^{\prime}\left(\Pi_{i=1}^{M} J_{M-t}\right)\right)=\ln \nu_{i}\left(\mathbf{T}_{M}^{\prime} \mathbf{T}_{M}\right) .
$$

Here, we used the matrix norm inequality $\left|\nu_{1}\left(A^{\prime} A\right)\right|\left|\nu_{1}\left(B^{\prime} B\right)\right| \geq\left|\nu_{1}\left((A B)^{\prime}(A B)\right)\right|$. Using this relationship, we can bound the largest Lyapunov exponent from above by $\bar{\lambda} \equiv \lim _{M \rightarrow \infty} \frac{1}{2 M} \sum_{t=1}^{M} \ln \nu_{1}\left(J_{M-t}^{\prime} J_{M-t}\right)$. We can consistently estimate this quantity, using its sample analogue,

$$
\widehat{\bar{\lambda}}_{M} \equiv \frac{1}{2 M} \sum_{t=1}^{M} \ln \nu_{1}\left(\widehat{J}_{M-t}^{\prime} \widehat{J}_{M-t}\right) .
$$

Corollary 3. Suppose that assumptions in Lemma 1 hold, $F_{i t}$ in $A 5$ is replaced by $F_{t}=$ $\partial \ln \nu_{1}\left(J_{t}^{\prime} J_{t}\right) / \partial \Delta \theta\left(Z_{t}\right), \eta_{i t}$ in $A 6$ is replaced by $\eta_{t}=\frac{1}{2} \ln \nu_{1}\left(J_{t-1}^{\prime} J_{t-1}\right)-\bar{\lambda}$. If $M=O\left([T / \log T]^{1 /(2+4 \phi)}\right)$, then

$$
\sqrt{M}\left(\widehat{\bar{\lambda}}_{M}-\bar{\lambda}\right) \Rightarrow N(0, \Phi) .
$$

Remark. For the multidimensional case, $\bar{\lambda}$ is always positive. This implies that the asymptotic distribution of the upper bound estimator seems to be useful only if the data is generated from a chaotic process (or $\lambda$ is positive). For example, when some specific positive value of the Lyapunov exponent is predicted by a theory, upper bound estimates below this value provide strong evidence against the hypothesis.

\section{Simulation results}

\subsection{Logistic map}

Since the testing procedure proposed in the previous section is based on asymptotic theory, it is of interest to examine its performance with sample sizes that are typical for economic time series. This section reports the result of the Monte Carlo experiments designed to assess the small sample performance of neural network estimates of Lyapunov exponent with various data generating processes.

We first examine the logistic map with system noise:

$$
X_{t}=a X_{t-1}\left(1-X_{t-1}\right)+\sigma \varepsilon_{t},
$$


where $\varepsilon_{t} / v_{t} \sim U(-1,1)$ independent of $X_{t}$, and

$$
v_{t}=\min \left\{a X_{t-1}\left(1-X_{t-1}\right), 1-a X_{t-1}\left(1-X_{t-1}\right)\right\}
$$

This particular form of heteroskedasticity ensures that the process $X_{t}$ is restricted to the unit interval. It is interesting to note that this simple one-dimensional model contains both a globally stable case $(0<a<3)$ and a chaotic case $(3.57<a \leq 4)$ depending on the parameter $a$. We use $a=1.5$ as an example with a negative Lyapunov exponent $(\lambda=-\ln 2$ when $\sigma=0)$ and $a=4$ as an example with a positive Lyapunov exponent $(\lambda=\ln 2$ when $\sigma=0)$.

For the neural network estimation, we use FUNFITS program developed by Nychka et al. (1996). As an activation function $\psi$, this program uses a type of sigmoid function

$$
\psi(u)=\frac{u(1+|u / 2|)}{2+|u|+u^{2} / 2},
$$

which was also employed by Nychka et al. (1992). For the estimation of $\Phi$, Bartlett's kernel $w(u)=$ $1-|u|$ with one lag is employed. We use the block subsample and equally spaced subsample in addition to the entire sample. To see how the results differ with the choice of the lags of the autoregression, we consider the cases with lag length $d$ varying from 1 to 4 . The results are based on the parameters $r=4, \sigma=0.25, T=200$ with 1000 replications. For subsample estimation, we use $M=66$ giving three blocks and estimates for each replication. The results are reported in Table 1 . When correct lag length is chosen $(d=1)$, the mean and the median of Lyapunov exponent estimates appeared close to the true value for both stable $(a=1.5)$ and chaotic $(a=4)$ cases. This suggests that our method works well even in the small sample environment. When $d$ increases, the number of estimates with incorrect sign increases for the stable case, while the estimates is robust to the additional lag lengths for the chaotic case. For the standard errors, there is a systematic downward bias for the stable case, but those for the chaotic case are in close agreement with actual standard deviations. Figures 1 and 2 show the finite sample densities of the Lyapunov exponent estimates standardized by the mean and variance superimposed on the standard normal densities. ${ }^{11}$ The distribution has some skewness, but with this small sample situation, it is close enough to normality predicted by the theory.

\subsection{Henon map}

Next we consider the example with a higher dimension. We employ a second-order chaotic process, the Henon map with system noise:

$$
X_{t}=1-a X_{t-1}^{2}+Y_{t-1}+\sigma \varepsilon_{t}, \quad Y_{t}=b X_{t-1}
$$

\footnotetext{
${ }^{11}$ Sample density is computed by kernel method with Silverman's rule of thumb bandwidth.
} 
with $a=1.4$ and $b=0.3$. By using the arguments similar to those of the logistic map example, we employ $\varepsilon_{t} / v_{t} \sim U(-1,1)$ with

$$
v_{t}=1.5-\left|1-1.4 X_{t-1}^{2}+Y_{t-1}\right|
$$

The (largest) Lyapunov exponent is known to be positive $(\lambda=0.408)$ in this model. This example is almost identical to the one used by Dechert and Gençay (1992). Following their experiments, we chose the parameter $\sigma$ so that the noise-to-signal ratio (defined with respect to standard deviation) is 0.005. Other numbers in the experiment are identical to those in our first example with logistic map. The simulation results are reported in Table 2 . When insufficient lag length $(d=1)$ is chosen, there is a slight downward bias. However, with sufficient lag length $(d \geq 2)$, the estimates are close to the true value. In addition, the standard errors capture the actual standard deviations reasonably well. Figure 3 shows the standardized finite sample density of Lyapunov exponent estimates and the standard normal density. Again, two densities are close, while there is a small skewness in the sample density.

\subsection{Barnett competition data}

Powerful properties of the neural network approach were confirmed by the successful results in the single-blind controlled competition conducted by William Barnett. Detail of the competition design and the results can be found in Barnett et al. (1997). However, since they used only point estimates of the neural network approach, it is of interest to examine how statistical procedure in this paper works for the same data used in the competition. ${ }^{12}$

The competition used two different sample sizes, 380 and 2000. Both small sample data and large sample data are taken from a single observation generated from the following five different models with $u_{t}$ being an iid standard normal random variable.

- Model I (Logistic map): $y_{t}=3.57 y_{t-1}\left(1-y_{t-1}\right)$ with $y_{0}=0.7$.

- Model II (GARCH): $y_{t}=h_{t}^{1 / 2} u_{t}$ where $h_{t}=1+0.1 y_{t-1}^{2}+0.8 h_{t-1}$ with $h_{0}=1$ and $y_{0}=0$.

- Model III (NLMA): $y_{t}=u_{t}+0.8 u_{t-1} u_{t-2}$.

- Model IV $(\mathrm{ARCH}): y_{t}=\left(1+0.5 y_{t-1}^{2}\right)^{1 / 2} u_{t}$ with $y_{0}=0$.

- Model V (ARMA): $y_{t}=0.8 y_{t-1}+0.15 y_{t-2}+u_{t}+0.3 u_{t-1}$ with $y_{0}=1$ and $y_{1}=0.7$.

\footnotetext{
${ }^{12}$ The data is downloaded from the archive given in Barnett et al. (1997, footnote 2).
} 
Of the five models described above, only Model I has a positive Lyapunov exponent. For this subsection and the empirical part of this paper, the number of lag length $(d)$ and the number of hidden units $(r)$ will be jointly determined by minimizing the BIC criterion (Schwarz, 1978) defined by

$$
B I C(d, r)=\ln \widehat{\sigma}^{2}+\frac{\ln T}{T}[1+r(d+2)]
$$

where $\widehat{\sigma}^{2}=T^{-1} \sum_{t=1}^{T}\left(X_{t}-\widehat{\theta}\left(X_{t-1}, \ldots, X_{t-d}\right)\right)^{2}$. For the HAC estimation required for the standard error, we employ the QS kernel with optimal bandwidth selection method developed in Andrews (1991). The employed block length $(M)$ for the small sample data $(T=380)$ is 70 giving a total of 5 blocks, while that for the large sample data $(T=2000)$ is 86 giving a total of 23 blocks.

The results for Barnett competition data are presented in Table 3. For the subsample estimates, the median values with rejection frequencies at $1 \%$ level of significance are reported. The results can be summarized as follows. First, the signs of all point estimates correspond to the true signs of the processes. Second, for models II to V, the positivity hypothesis is rejected at $1 \%$ level based on full sample estimation. Except for block subsample based estimates for model V, the same hypothesis is rejected for all estimates in each model from II to V. These results confirm the validity of the neural network approach and our testing procedure. Third, positivity of the Lyapunov exponent in model I is not rejected for both full sample and subsample cases, but it did not provide strong evidence against the negativity.

\section{Application to financial data}

Over the past decades, numerous models that can generate chaos in economic variables have been developed. For example, Brock and Hommes (1998) showed that chaos in stock price was possible if heterogeneous beliefs of agents were introduced in traditional asset pricing model. ${ }^{13}$ In this section, we apply our proposed procedure to investigate the possibility of chaos in the U.S. financial market using stock price series. ${ }^{14}$

We use daily observations on the Dow Jones Industrial Average (DJIA). Sample period is from January 3, 1928 to October 18, 2000 giving a total of 18,490 observations. It should be noted that the period of the stock market crash of 1987 is included in the sample period. The stock return is simply defined as the difference of $\log$ of the stock price index $\left(R_{t}=\Delta \ln P_{t}\right)$. Following Taylor's (1986) finding, it is now well-known that the absolute return $\left(\left|R_{t}\right|\right)$ has higher autocorrelation compared

\footnotetext{
${ }^{13}$ See Abhyankar, Copeland and Wong (1997) for a survey of previous results of analyses of chaos using financial data.

${ }^{14}$ Other economic theories predict chaos in real aggregate series. The method proposed in this paper is also applied to international real output series by Shintani and Linton (2001).
} 
to the return series $\left(R_{t}\right)$. Ding, Granger and Engle (1993) also examined the correlation of power transformation of the absolute return $\left(\left|R_{t}\right|^{k}\right)$ and found quite high autocorrelations. Extending this line of approach, we estimate the Lyapunov exponent of various power transformed absolute return series. Table 4 shows the sample autocorrelations of the transformed absolute DJIA stock returns $\left|R_{t}\right|^{k}$ for $k=0.5,1,1.5,2,2.5$ in addition to those of the untransformed return series. The return series has small positive first order autocorrelation and small negative second order autocorrelation, while the transformed absolute return has much higher autocorrelations with $k=1$ being the highest. These results are very similar to those of Ding, Granger and Engle (1993) based on S\&P 500 series with number of observation close to that of our data.

The estimated Lyapunov exponents for each series is presented in Table 5 along with the $t$ statistics and $p$-values for the null hypothesis of positive Lyapunov exponent $\left(H_{0}: \lambda \geq 0\right)$. The block length $(M)$ and the number of blocks used for subsampling estimates are 127 and 145, respectively. The number of hidden units $(r)$ are selected using BIC. For all cases, the Lyapunov exponents from full sample estimation are negative, and the positivity hypothesis is significantly rejected at the $1 \%$ level with exception of transformed series with $k=2.5$. Similar strong evidence is obtained from subsample estimation except for the same series. Another interesting observation is that the Lyapunov exponents are larger for the transformed absolute returns than for the level of returns, suggesting less stability in volatility (or absolute values) than in returns themselves. These results from various transformed data offer strong statistical evidence against the chaotic explanation in stock returns. This strengthens the results in Abhyankar, Copeland and Wong (1997) who obtained negative Lyapunov exponent point estimates for both S\&P500 cash and futures series with 5-minute and 1-minute frequencies.

\section{Conclusion}

This paper derived the asymptotic distribution of the neural network Lyapunov exponent estimator proposed by Nychka et al. (1992) and others and introduced a formal statistical framework of testing hypotheses concerning the sign of the Lyapunov exponent. Such a procedure offers a useful empirical tool for detecting chaos in a noisy system. The small sample properties of the new procedure were examined in simulations, which indicate that the performance of the procedure is satisfactory in moderate-sized samples. The procedure was applied to investigate chaotic behavior of financial market. In most cases we strongly rejected the hypothesis of chaos in the stock return series with one mild exception in some higher power transformed absolute returns. 


\section{Appendix}

\section{Proof of Lemma 1}

In this proof, we define the (weighted) $L_{p}$ space (with distribution function $F(x)$ ) by the set of $L_{p^{-}}$ integrable functions with norm $\|g\|_{p}=\left\{\int_{\chi}|g(x)|^{p} d F(x)\right\}^{1 / p}$ and associated metric from this norm. For $p=\infty$, we use $\|g\|_{\infty}=\sup _{x \in \chi}|g(x)|$. We will denote $L_{2}$ norm $\|g\|_{2}$ simply by $\|g\|$. Similarly, we define the (weighted) Sobolev $W_{p}^{m}$ space with set of functions with $L_{p}$-integrable (partial) derivatives up to order $m$ with norm $\|g\|_{m, p}=\left\{\sum_{|\mu|=0}^{m} \int_{\chi}\left|D^{\mu} g(x)\right|^{p} d F(x)\right\}^{1 / p}$ and associated metric from this norm. For $p=\infty$, we use $\|g\|_{m, \infty}=\max _{0 \leq|\mu| \leq m} \sup _{x \in \chi}\left|D^{\mu} g(x)\right|$.

(a) To simplify the argument, we first derived the result for one-dimensional case, and then extend the result to the multidimensional case. For $d=1$, we denote $\mathcal{Z}=\chi$ and our goal is to obtain the convergence rate for

$$
\sup _{x \in \chi}\left|D \widehat{\theta}(x)-D \theta_{0}(x)\right| .
$$

Note that interpolation inequality (See Gabushin, 1967, and Shen and Wong, 1994) implies

$$
\left\|g(x)-g_{0}(x)\right\|_{\infty} \leq K\left\|g(x)-g_{0}(x)\right\|^{2(m-1) / 2 m}\left\|D^{m} g(x)-D^{m} g_{0}(x)\right\|^{1 / 2 m} .
$$

where $K$ is a fixed constant. Substituting $g(x)=D \widehat{\theta}(x), g_{0}(x)=D \theta_{0}(x), m=1$ yields

$$
\left\|D \widehat{\theta}(x)-D \theta_{0}(x)\right\|_{\infty} \leq K\left\|D \widehat{\theta}(x)-D \theta_{0}(x)\right\|^{1 / 2}\left\|D^{2} \widehat{\theta}(x)-D^{2} \theta_{0}(x)\right\|^{1 / 2}
$$

If we use that

$$
\left\|\widehat{D \theta}(x)-D \theta_{0}(x)\right\| \leq\left\|\widehat{\theta}(x)-\theta_{0}(x)\right\|_{1,2} \leq\left\|\widehat{\theta}(x)-\theta_{0}(x)\right\|_{2,2}
$$

and

$$
\left\|D^{2} \widehat{\theta}(x)-D^{2} \theta_{0}(x)\right\| \leq\left\|\widehat{\theta}(x)-\theta_{0}(x)\right\|_{2,2},
$$

the $\left\|D \widehat{\theta}(x)-D \theta_{0}(x)\right\|_{\infty}$ term is bounded by $K\left\|\widehat{\theta}(x)-\theta_{0}(x)\right\|_{2,2}$. Therefore it suffices to show the convergence rate of $\left\|\hat{\theta}(x)-\theta_{0}(x)\right\|_{2,2}$.

Approximation rate in Sobolev norm is derived in Hornik et al. (1994). Convergence rate of the estimator in $L_{2}$ norm is derived in Chen and Shen (1998) and Chen and White (1999). We will combine their results to derive convergence rate of estimator in Sobolev norm. From the definition of criterion in Assumption B1(a), we have

$$
E\left[l\left(Z_{t}, \theta\right)-l\left(Z_{t}, \theta_{0}\right)\right]=\frac{1}{2}\left\|\theta-\theta_{0}\right\|^{2} .
$$


Since Assumption A3 implies the boundedness of the third derivatives, equivalence of $L_{2}$ norm and Sobolev norm with second derivatives holds and there exist two constants $c_{1}$ and $c_{2}$ satisfying

$$
c_{1}\left\|\theta-\theta_{0}\right\|_{2,2}^{2} \leq E\left[l\left(Z_{t}, \theta\right)-l\left(Z_{t}, \theta_{0}\right)\right] \leq c_{2}\left\|\theta-\theta_{0}\right\|_{2,2}^{2}
$$

which is required for Theorem 1 in Chen and Shen (1998). Further, Condition A1 in Chen and Shen can be replaced by our class of mixing condition in assumption A1(a) which is shown by Chen and White (1999). Condition A2 and A4 in Chen and Shen (Assumption 3.4 (a) and (b) in Chen and White) follows from the proof of Proposition 1 in Chen and Shen. Therefore, from Theorem 1 in Chen and Shen (1998), we have

$$
\left\|\widehat{\theta}_{T}-\theta_{0}\right\|_{2,2}=O_{p}\left(\max \left(\delta_{T},\left\|\theta_{0}-\pi_{T} \theta_{0}\right\|_{2,2}\right)\right)
$$

where $\pi_{T} \theta_{0} \in \Theta_{T}$ and

$$
\delta_{T}=\inf \left\{\delta>0: \delta^{-2} \int_{\delta^{2}}^{\delta}\left[H\left(\varepsilon, \mathcal{F}_{T}\right)\right]^{1 / 2} d \varepsilon \leq \text { const. } \times n^{1 / 2}\right\}
$$

where $H\left(\varepsilon, \mathcal{F}_{T}\right)$ is the $L_{2}$ metric entropy with bracketing which controls the size of the space of criterion differences induced by $\theta \in \Theta_{T}$ (See Chen and Shen, 1998, for the definition. Formally, the bracketing $L_{2}$ metric entropy of the space of the $L_{2}$ measurable functions indexed by $\Theta_{T}$ given by $\mathcal{F}_{T}=\left\{h(\theta, z)=l(\theta, z)-l\left(\theta_{0}, z\right): \theta \in \Theta_{T}\right\}$ is defined as follows. For any given $\varepsilon$, if there exists $S(\varepsilon, N)=\left\{h_{1}^{l}, h_{1}^{u}, \ldots h_{N}^{l}, h_{N}^{u}\right\} \subset L_{2}$ with $\max _{1 \leq j \leq N}\left\|h_{j}^{u}-h_{j}^{l}\right\| \leq \varepsilon$ such that for any $h \in \mathcal{F}_{T}$ there exists a $j$ with $h_{j}^{l} \leq h \leq-h_{j}^{u}$ a.e., then $S(\varepsilon, N)$ is called a bracketing $\varepsilon$-covering of $\mathcal{F}_{T}$ with respect to $\|\cdot\|$. We define $H\left(\varepsilon, \mathcal{F}_{T}\right)$ by $\log (\min \{N: S(\varepsilon, N)\})$.)

Using the result in the proof of Theorem 3.1 in Chen and White (1999), we have

$$
H\left(\varepsilon, \mathcal{F}_{T}\right) \leq 2^{k} r B_{T}(d+1) \log \left(2^{k} r B_{T}(d+1) / \varepsilon\right)
$$

and

$$
\delta_{T}=\text { const. } \times[r \log (r)]^{1 / 2} T^{-1 / 2} .
$$

From Hornik et al. (1994), the approximation rate in Sobolev $W_{2}^{2}$ norm is given by

$$
\left\|\theta_{0}-\pi_{T} \theta_{0}\right\|_{2,2} \leq \text { const. } \times r^{-1 / 2} .
$$

By choosing $\delta_{T}=\left\|\theta_{0}-\pi_{T} \theta_{0}\right\|_{2,2}$, we have

$$
r^{2} \log r=O(T)
$$

and

$$
\left\|\widehat{\theta}_{T}-\theta_{0}\right\|_{2,2}=O_{p}\left([T / \log T]^{-1 / 4}\right) .
$$


as required.

(b) For the multidimensional case, from Gabushin's interpolation inequality, we have

$$
\left\|\Delta \widehat{\theta}_{i}(z)-\Delta \theta_{i 0}(z)\right\|_{\infty} \leq K\left\|\Delta \widehat{\theta}_{i}(z)-\Delta \theta_{i 0}(z)\right\|^{1 / 2}\left\|\Delta^{2} \widehat{\theta}_{i}(z)-\Delta^{2} \theta_{i 0}(z)\right\|^{1 / 2} .
$$

for each $i=1, \ldots, d$ with $|\cdot|$ here being absolute value. If we use that

$$
\sum_{i=1}^{d}\left\|\Delta \widehat{\theta}_{i}(z)-\Delta \theta_{i 0}(z)\right\| \leq\left\|\widehat{\theta}(z)-\theta_{0}(z)\right\|_{1,2} \leq\left\|\widehat{\theta}(z)-\theta_{0}(z)\right\|_{2,2}
$$

and

$$
\sum_{i=1}^{d}\left\|\Delta^{2} \widehat{\theta}_{i}(z)-\Delta^{2} \theta_{i 0}(z)\right\| \leq\left\|\widehat{\theta}(z)-\theta_{0}(z)\right\|_{2,2}
$$

then,

$$
\begin{aligned}
\sup _{z \in \mathcal{Z}}\left|\Delta \widehat{\theta}(z)-\Delta \theta_{0}(z)\right| & =\sup \sum_{i=1}^{d}\left|\Delta \widehat{\theta}_{i}(z)-\Delta \theta_{i 0}(z)\right| \\
& \leq \sum_{i=1}^{d} \sup \left|\Delta \widehat{\theta}_{i}(z)-\Delta \theta_{i 0}(z)\right| \\
& =\sum_{i=1}^{d}\left\|\Delta \widehat{\theta}_{i}(z)-\Delta \theta_{i 0}(z)\right\|_{\infty} \\
& \leq K \sum_{i=1}^{d}\left(\left\|\Delta \widehat{\theta}_{i}(z)-\Delta \theta_{i 0}(z)\right\|^{1 / 2}\left\|\Delta^{2} \widehat{\theta}_{i}(z)-\Delta^{2} \theta_{i 0}(z)\right\|^{1 / 2}\right) \\
& \leq K\left(\sum_{i=1}^{d}\left\|\Delta \widehat{\theta}_{i}(z)-\Delta \theta_{i 0}(z)\right\|\right)^{1 / 2}\left(\sum_{i=1}^{d}\left\|\Delta^{2} \widehat{\theta}_{i}(z)-\Delta^{2} \theta_{i 0}(z)\right\|\right)^{1 / 2} \\
& \leq K\left\|\widehat{\theta}(z)-\theta_{0}(z)\right\|_{2,2}
\end{aligned}
$$

where the second inequality follows from Cauchy-Schwarz's inequality. Therefore it again suffices to show the convergence rate of $\left\|\widehat{\theta}(z)-\theta_{0}(z)\right\|_{2,2}$. Since the convergence rate of neural network estimator does not depend on $d$, the same argument for the one-dimensional case can be directly applied and the result follows.

\section{Proof of Lemma 2}

Similar to the proof of Lemma 1 it suffices to show the convergence rate of $\left\|\widehat{\theta}(x)-\theta_{0}(x)\right\|_{2,2}$ for the one-dimensional case.

Since additional assumption B3 is identical to assumption $\mathrm{H}$ in Chen and White, the result for the improved rate in Sobolev norm in Theorem 2.1 of Chen and White can be used. 
The improved approximation rate in Sobolev $W_{2}^{2}$ norm is now given by

$$
\left\|\theta_{0}-\pi_{T} \theta_{0}\right\|_{2,2} \leq \text { const. } \times r^{-1 / 2-\alpha / d^{*}} .
$$

From

$$
\delta_{T}=\text { const. } \times[r \log (r)]^{1 / 2} T^{-1 / 2} .
$$

with choice of $\delta_{T}=\left\|\theta_{0}-\pi_{T} \theta_{0}\right\|_{2,2}$, we have

$$
r^{2\left(1+\alpha / d^{*}\right)} \log r=O(T)
$$

and

$$
\left\|\widehat{\theta}_{T}-\theta_{0}\right\|_{2,2}=O_{p}\left([T / \log T]^{-\frac{1+\left(2 \alpha / d^{*}\right)}{4\left(1+\left(\alpha / d^{*}\right)\right)}}\right)=o_{p}\left(T^{-1 / 4}\right) .
$$

as required. The same argument can be used for multidimensional case as in the proof of Lemma 1.

\section{Proof of Theorem 1}

By rearranging terms,

$$
\sqrt{M}\left(\widehat{\lambda}_{M}-\lambda\right)=\sqrt{M}\left(\widehat{\lambda}_{M}-\lambda_{M}\right)+\sqrt{M}\left(\lambda_{M}-\lambda\right) .
$$

For the second term, we have

$$
\sqrt{M}\left(\lambda_{M}-\lambda\right)=\frac{1}{2 \sqrt{M}} \sum_{t=1}^{M}\left[\ln \left(D \theta_{0}\left(X_{t-1}\right)\right)^{2}-2 \lambda\right] \Rightarrow N(0, \Phi)
$$

by the CLT of Herrndorf (1984, Corollary 1) and A5*.

For the first term,

$$
\begin{aligned}
\left|\sqrt{M}\left(\widehat{\lambda}_{M}-\lambda_{M}\right)\right|= & \left|\frac{1}{2 \sqrt{M}} \sum_{t=1}^{M}\left[\ln \left(D \widehat{\theta}\left(X_{t-1}\right)\right)^{2}-\ln \left(D \theta_{0}\left(X_{t-1}\right)\right)^{2}\right]\right| \\
= & \left|\frac{1}{\sqrt{M}} \sum_{t=1}^{M} \frac{1}{D \theta^{*}\left(X_{t-1}\right)}\left[D \widehat{\theta}\left(X_{t-1}\right)-D \theta_{0}\left(X_{t-1}\right)\right]\right| \\
\leq & {[T / \log T]^{-\frac{1}{4}} M^{\frac{1}{2}+\phi}\left[[T / \log T]^{\frac{1}{4}} \sup _{x \in \chi}\left|D \widehat{\theta}(x)-D \theta_{0}(x)\right|\right] } \\
& \times\left(\frac{1}{M^{\phi} \min _{\left\{t: X_{t} \in \chi\right\}}\left|D \theta^{*}(x)\right|}\right)=o_{p}(1)
\end{aligned}
$$

where the second equality holds by a one-term Taylor expansion about $D \theta_{0}\left(X_{t-1}\right)$ (with $D \theta^{*}\left(X_{t-1}\right)$ lying between $D \theta_{0}\left(X_{t-1}\right)$ and $\left.D \widehat{\theta}\left(X_{t-1}\right)\right)$. The convergence to zero holds because of $[T / \log T]^{-\frac{1}{4}} M^{\frac{1}{2}+\phi}=$ $O(1)$ from the growth rate of block length, uniform convergence from Lemma 1 and $\left(M^{\phi} \min _{1 \leq t \leq M} \mid D \theta^{*}\left(X_{t}\right.\right.$ $O_{p}(1)$ from $\mathrm{A} 4^{*}$, respectively. The latter can be verified by using the argument given in the proof of Theorem 1 in Whang and Linton (1999). 


\section{Proof of Theorem 2}

By rearranging terms,

$$
\sqrt{M}\left(\widehat{\lambda}_{i M}-\lambda_{i}\right)=\sqrt{M}\left(\widehat{\lambda}_{i M}-\lambda_{i M}\right)+\sqrt{M}\left(\lambda_{i M}-\lambda_{i}\right)
$$

where

$$
\lambda_{i M}=\frac{1}{2 M} \ln \nu_{i}\left(\left(\Pi_{t=1}^{M} J_{M-t}\right)^{\prime}\left(\Pi_{t=1}^{M} J_{M-t}\right)\right) .
$$

For the second term, we have

$$
\begin{aligned}
\sqrt{M}\left(\lambda_{i M}-\lambda_{i}\right) & =\sqrt{M}\left[\frac{1}{2 M} \ln \nu_{i}\left(\left(\Pi_{t=1}^{M} J_{M-t}\right)^{\prime}\left(\Pi_{t=1}^{M} J_{M-t}\right)\right)-\lambda_{i}\right] \\
& =\sqrt{M}\left[\frac{1}{2 M} \ln \nu_{i}\left(\mathbf{T}_{M}^{\prime} \mathbf{T}_{M}\right)-\lambda_{i}\right] \\
& =\sqrt{M}\left[\frac{1}{2 M} \ln \left(\frac{\nu_{i}\left(\mathbf{T}_{M}^{\prime} \mathbf{T}_{M}\right)}{\nu_{i}\left(\mathbf{T}_{M-1}^{\prime} \mathbf{T}_{M-1}\right)}\right)+\frac{1}{2 M} \ln \nu_{i}\left(\mathbf{T}_{M-1}^{\prime} \mathbf{T}_{M-1}\right)-\lambda_{i}\right] \\
& =\sqrt{M}\left[\sum_{k=1}^{M-1} \frac{1}{2 M} \ln \left(\frac{\nu_{i}\left(\mathbf{T}_{M-k+1}^{\prime} \mathbf{T}_{M-k+1}\right)}{\nu_{i}\left(\mathbf{T}_{M-k}^{\prime} \mathbf{T}_{M-k}\right)}\right)+\frac{1}{2 M} \ln \nu_{i}\left(\mathbf{T}_{1}^{\prime} \mathbf{T}_{1}\right)-\lambda_{i}\right] \\
& =\sqrt{M}\left[\frac{1}{M} \sum_{k=1}^{M} \xi_{i, M-k+1}-\lambda_{i}\right] \\
& =\frac{1}{\sqrt{M}} \sum_{t=1}^{M}\left[\xi_{i t}-\lambda_{i}\right] \Rightarrow N\left(0, \Phi_{i}\right)
\end{aligned}
$$

by the CLT of Herrndorf (1984, Corollary 1) and results of Furstenberg and Kesten (1960, Theorem 3) and A5.

For the first term,

$$
\begin{aligned}
\left|\sqrt{M}\left(\widehat{\lambda}_{i}-\lambda_{i M}\right)\right|= & \frac{1}{2 \sqrt{M}}\left|\ln \nu_{i}\left(\left(\Pi_{t=1}^{M} \widehat{J}_{M-t}\right)^{\prime}\left(\Pi_{t=1}^{M} \widehat{J}_{M-t}\right)\right)-\ln \nu_{i}\left(\left(\Pi_{t=1}^{M} J_{M-t}\right)^{\prime}\left(\Pi_{t=1}^{M} J_{M-t}\right)\right)\right| \\
= & \left|\frac{1}{\sqrt{M}} \sum_{t=1}^{M} F_{i, t-1}\left(J_{M-1}^{*}, \ldots, J_{0}^{*}\right)^{\prime}\left[\Delta \widehat{\theta}\left(Z_{t-1}\right)-\Delta \theta_{0}\left(Z_{t-1}\right)\right]\right| \\
\leq & {[T / \log T]^{-\frac{1}{4}} M^{\frac{1}{2}+\phi}\left[[T / \log T]^{\frac{1}{4}} \sup _{z \in \mathcal{Z}}\left|\Delta \widehat{\theta}(z)-\Delta \theta_{0}(z)\right|\right] } \\
& \times M^{-\phi} \max _{1 \leq t \leq M}\left|F_{i, t-1}\left(J_{M-1}^{*}, \ldots, J_{0}^{*}\right)\right|=o_{p}(1)
\end{aligned}
$$

where the second equality follows from a one-term Taylor expansion

$$
\begin{aligned}
& \ln \nu_{i}\left(\left(\Pi_{t=1}^{M} \widehat{J}_{M-t}\right)^{\prime}\left(\Pi_{t=1}^{M} \widehat{J}_{M-t}\right)\right) \\
= & \ln \nu_{i}\left(\left(\Pi_{t=1}^{M} J_{M-t}\right)^{\prime}\left(\Pi_{t=1}^{M} J_{M-t}\right)\right)+\frac{\partial \ln \nu_{i}\left(\left(\Pi_{t=1}^{M} J_{M-t}^{*}\right)^{\prime}\left(\prod_{t=1}^{M} J_{M-t}^{*}\right)\right)}{\partial \Delta \theta_{0}\left(Z_{t-1}\right)^{\prime}}\left[\Delta \widehat{\theta}\left(Z_{t-1}\right)-\Delta \theta_{0}\left(Z_{t-1}\right)\right] \\
= & \ln \nu_{i}\left(\left(\Pi_{t=1}^{M} J_{M-t}\right)^{\prime}\left(\Pi_{t=1}^{M} J_{M-t}\right)\right)+F_{i, t-1}\left(J_{M-1}^{*}, \ldots, J_{0}^{*}\right)^{\prime}\left[\Delta \widehat{\theta}\left(Z_{t-1}\right)-\Delta \theta_{0}\left(Z_{t-1}\right)\right]
\end{aligned}
$$


where the elements of $J_{t}^{*}$ lie between those of $\widehat{J}_{t}$ and $J_{t}$ for $t=0, \ldots, M-1$. By analogous to the proof of Theorem 1, the convergence to zero holds because of $[T / \log T]^{-\frac{1}{4}} M^{\frac{1}{2}+\phi}=O(1)$ from the growth rate of block length, uniform convergence from Lemma 1 and $M^{-\phi} \max _{1 \leq t \leq M}\left|F_{i, t-1}\left(J_{M-1}^{*}, \ldots, J_{0}^{*}\right)\right|=O_{p}(1)$ from A4, respectively.

\section{Proof of Corollary 1}

We only prove the one-dimensional case since the multidimensional case can be obtained using the similar argument. First define

$$
\widetilde{\Phi}=\sum_{j=-M+1}^{M-1} w\left(j / S_{M}\right) \widetilde{\gamma}(j) \text { and } \widetilde{\gamma}(j)=\frac{1}{M} \sum_{t=|j|+1}^{M} \eta_{t} \eta_{t-|j|}
$$

where $\eta_{t}=\left\{\ln \left|D \theta\left(X_{t-1}\right)\right|-\lambda\right\}$. From Proposition 1 of Andrews (1991), $\widetilde{\Phi} \stackrel{p}{\rightarrow} \Phi$. Therefore, it suffices to show that $\widehat{\Phi} \stackrel{p}{\rightarrow} \widetilde{\Phi}$. Since $S_{M}^{2} / M \rightarrow 0$, the result follows by showing

$$
\begin{aligned}
& \frac{\sqrt{M}}{S_{M}}|\widehat{\Phi}-\widetilde{\Phi}|=\frac{\sqrt{M}}{S_{M}}\left|\sum_{j=-M+1}^{M-1} w\left(\frac{j}{S_{M}}\right)\{\widehat{\gamma}(j)-\widetilde{\gamma}(j)\}\right| \\
\leq & \sqrt{M} \sup _{j}|\widehat{\gamma}(j)-\widetilde{\gamma}(j)|\left(\frac{1}{S_{M}} \sum_{j=-M+1}^{M-1}\left|w\left(\frac{j}{S_{M}}\right)\right|\right)=O_{p}(1) .
\end{aligned}
$$

The second element is bounded since $\left(1 / S_{M}\right) \sum_{j=-M+1}^{M-1}\left|w\left(j / S_{M}\right)\right| \rightarrow \int_{-\infty}^{\infty}|k(x)| d x<\infty$. For the first element, we have

$$
\begin{aligned}
& \sqrt{M} \sup _{j}|\widehat{\gamma}(j)-\gamma(j)|=\sqrt{M} \sup _{j}\left|\frac{1}{T} \sum_{t=|j|+1}^{T}\left(\widehat{\eta}_{t} \widehat{\eta}_{t-|j|}-\eta_{t} \eta_{t-|j|}\right)\right| \\
& \leq \sqrt{M} 2\left(\sup _{x \in \chi}\left|D \widehat{\theta}(x)-D \theta_{0}(x)\right|\right)\left[\frac{1}{T} \sum_{t=1}^{T} \sup _{\theta \in \Theta} \frac{1}{\left|D \theta^{*}\left(X_{t-1}\right)\right|^{2}}\right]^{1 / 2}\left[\frac{1}{T} \sum_{t=1}^{T} \sup _{\theta \in \Theta} \eta_{t}^{2}\right]^{1 / 2} \\
& +\sqrt{M}\left(\sup _{x \in \chi}\left|D \widehat{\theta}(x)-D \theta_{0}(x)\right|^{2}\right)\left[\frac{1}{T} \sum_{t=1}^{T} \sup _{\theta \in \Theta} \frac{1}{\left|D \theta^{*}\left(X_{t-1}\right)\right|^{2}}\right] \\
& =\left\{2[T / \log T]^{-\frac{1}{4}} M^{\frac{1}{2}+\phi} \frac{[T / \log T]^{\frac{1}{4}} \sup _{x \in \chi}\left|D \widehat{\theta}(x)-D \theta_{0}(x)\right|}{M^{\phi} \min _{\left\{t: X_{t-1} \in \chi\right\}}\left|D \theta^{*}\left(X_{t-1}\right)\right|}\left[\frac{1}{M} \sum_{t=1}^{M}\left(\ln \left|D \theta_{0}\left(X_{t-1}\right)\right|-\lambda\right)^{2}\right]^{1 / 2}\right. \\
& \left.+M^{-1 / 2} \times\left[[T / \log T]^{-\frac{1}{4}} M^{\frac{1}{2}+\phi}\right]^{2} \frac{[T / \log T]^{\frac{1}{2}} \sup _{x \in \chi}\left|D \widehat{\theta}(x)-D \theta_{0}(x)\right|^{2}}{M^{2 \phi} \min _{\left\{t: X_{t-1} \in \chi\right\}}\left|D \theta^{*}\left(X_{t-1}\right)\right|^{2}}\right\} O(1) \\
& =O_{p}(1)
\end{aligned}
$$


using $[T / \log T]^{-\frac{1}{4}} M^{\frac{1}{2}+\phi}=O(1)$ from condition in Theorem 1, uniform convergence from Lemma 1 and

$\left(M^{\phi} \min _{1 \leq t \leq M}\left|D \theta^{*}\left(X_{t-1}\right)\right|\right)^{-1}=O_{p}(1)$ from A4*, respectively.

\section{Proof of Corollary 2}

Since the proof is similar to the one for Theorem 1(a) in Whang and Linton (1999), we only provide the sketch of proof.

By rearranging terms,

$$
\sqrt{T}\left(\widehat{\lambda}_{T}-\lambda\right)=\sqrt{T}\left(\widehat{\lambda}_{T}-\lambda_{T}\right)+\sqrt{T}\left(\lambda_{T}-\lambda\right) .
$$

For the second term, we have asymptotics identical to those in Theorem 1. For the first term,

$$
\begin{aligned}
& \sqrt{T}\left(\widehat{\lambda}_{T}-\lambda_{T}\right) \\
= & \frac{1}{\sqrt{T}} \sum_{t=1}^{T} \frac{1}{D \theta_{0}\left(X_{t-1}\right)}\left[D \widehat{\theta}\left(X_{t-1}\right)-D \theta_{0}\left(X_{t-1}\right)\right] \\
& -\frac{1}{2 \sqrt{T}} \sum_{t=1}^{T} \frac{1}{\left[D \theta^{*}\left(X_{t-1}\right)\right]^{2}}\left[D \widehat{\theta}\left(X_{t-1}\right)-D \theta_{0}\left(X_{t-1}\right)\right]^{2} \\
= & \frac{1}{\sqrt{T}} \sum_{t=1}^{T} \frac{1}{D \theta_{0}\left(X_{t-1}\right)}\left[D \widehat{\theta}\left(X_{t-1}\right)-D \theta_{0}\left(X_{t-1}\right)\right]+o_{p}(1) \\
= & \sqrt{T} \int_{\chi} \frac{1}{D \theta_{0}(x)}\left[D \widehat{\theta}(x)-D \theta_{0}(x)\right] f(x) d x+o_{p}(1) \\
= & -\sqrt{T} \int_{\chi}\left[D\left(\frac{f(x)}{D \theta_{0}(x)}\right) \frac{1}{f(x)}\right]\left\{\widehat{\theta}(x)-\theta_{0}(x)\right\} f(x) d x+o_{p}(1) \\
= & \sqrt{T} \int_{\chi}^{v} v(x)\left\{\widehat{\theta}(x)-\theta_{0}(x)\right\} f(x) d x+o_{p}(1) \\
= & \sqrt{T}\left\langle v(x), \widehat{\theta}-\theta_{0}\right\rangle+o_{p}(1) \\
= & \frac{1}{\sqrt{T}} \sum_{t=1}^{T} l_{\theta_{0}}^{\prime}\left[v(x), X_{t-1}\right]+o_{p}(1) \\
= & \frac{1}{\sqrt{T}} \sum_{t=1}^{T} v\left(X_{t-1}\right) u_{t}+o_{p}(1) .
\end{aligned}
$$

The first equality follows from a two-term Taylor expansion about $D \theta_{0}\left(X_{t-1}\right)$ with $D \theta^{*}\left(X_{t-1}\right)$ lies between $D \theta_{0}\left(X_{t-1}\right)$ and $D \widehat{\theta}\left(X_{t-1}\right)$. The second equality follows from the fact that the second term is bounded by

$$
\frac{1}{2}\left[T^{\frac{1}{4}} \sup _{x \in \chi}\left|D \widehat{\theta}(x)-D \theta_{0}(x)\right|\right]^{2} \frac{1}{T} \sum_{t=1}^{T} \frac{1}{\left[D \theta^{*}\left(X_{t-1}\right)\right]^{2}}
$$




$$
\leq o_{p}(1) \times\left(\frac{1}{\min _{\left\{t: X_{t-1} \in \chi_{T}\right\}}\left|D \theta^{*}\left(X_{t-1}\right)\right|}\right)^{2}=o_{p}(1)
$$

where the inequality follows by the uniform consistency results in Lemma 2, the last convergence to zero holds because $\left(\min _{\left\{t: X_{t-1} \in \chi_{T}\right\}}\left|D \theta^{*}\left(X_{t-1}\right)\right|\right)^{-2}=O_{p}(1)$ by $\mathrm{A} 4^{*}$. The third equality follows from stochastic equicontinuity argument employed in Whang and Linton (1999). The forth equality follows from integration by parts with the zero boundary condition. The last three equalities follows from the definition of the linear functional $l_{\theta_{0}}^{\prime}\left[\widehat{\theta}-\theta_{0}, X_{t-1}\right]$ and inner product $\langle.,$.$\rangle used in Shen$ (1997), Chen and Shen (1998) and Chen and White (1999), and

$$
l_{\theta_{0}}^{\prime}\left[\widehat{\theta}-\theta_{0}, X_{t-1}\right]=\left[\widehat{\theta}-\theta_{0}\right] u_{t}
$$

from our criterion function given in $\mathrm{A} 3(\mathrm{a})$.

\section{Proof of Corollary 3}

We use a one-term Taylor expansion

$$
\begin{aligned}
& \ln \nu_{1}\left(\widehat{J}_{t-1}^{\prime} \widehat{J}_{t-1}\right) \\
= & \ln \nu_{1}\left(J_{t-1}^{\prime} J_{t-1}\right)+\frac{\partial \nu_{1}\left(J_{t-1}^{* \prime} J_{t-1}^{*}\right)}{\partial \Delta \theta_{0}\left(Z_{t-1}\right)^{\prime}}\left[\Delta \widehat{\theta}\left(Z_{t-1}\right)-\Delta \theta_{0}\left(Z_{t-1}\right)\right] \\
= & \ln \nu_{1}\left(J_{t-1}^{\prime} J_{t-1}\right)+F_{t-1}^{\prime}\left[\Delta \widehat{\theta}\left(Z_{t-1}\right)-\Delta \theta_{0}\left(Z_{t-1}\right)\right]
\end{aligned}
$$

where the elements of $J_{t-1}^{*}$ lie between those of $\widehat{J}_{t-1}$ and $J_{t-1}$. The result follows from the argument similar (but simpler) to the one used in the proof of Theorem 2 . 


\section{References}

Abhyankar, A., L. S. Copeland and W. Wong (1997). "Uncovering nonlinear structure in real-time stock-market indexes: The S\&P 500, the DAX, the Nikkei 225, and the FTSE-100." Journal of Business and Economic Statistics 15(1): 1-14.

Andrews, D. W. K. (1991). "Heteroskedasticity and autocorrelation consistent covariance matrix estimation." Econometrica 59(3): 817-858.

Bailey, B. A. (1996). Local Lyapunov exponents: Predictability depends on where you are. Nonlinear dynamics and economics, edited by W. A. Barnett, A. P. Kirman and M. Salmon. Cambridge, Cambridge University Press: 345-359.

Barnett, W. A., A. R. Gallant, M. J. Hinich, J. A. Jungeilges, D. T. Kaplan and M. J. Jensen (1995). "Robustness of nonlinearity and chaos tests to measurement error, inference method, and sample size." Journal of Economic Behavior and Organization 27: 301-320.

Barnett, W. A., A. R. Gallant, M. J. Hinich, J. A. Jungeilges, D. T. Kaplan and M. J. Jensen (1997). "A single-blind controlled competition among tests for nonlinearity and chaos." Journal of Econometrics 82: 157-192.

Barron, A. R. (1993). "Universal approximation bounds for superpositions of a sigmoidal function." IEEE Transactions on Information Theory 39(3): 930-945.

Brock, W. A., W. D. Dechert, J. A. Scheinkman and B. LeBaron (1996). "A test for independence based on the correlation dimension." Econometric Reviews 15(3): 197-235.

Brock, W. A. and C. H. Hommes (1998). "Heterogeneous beliefs and routes to chaos in a simple asset pricing model" Journal of Economic Dynamics and Control 22: 1235-1274.

Chen, X. and X. Shen (1998). "Sieve extremum estimates for weakly dependent data." Econometrica $66(2): 289-314$.

Chen, X. and H. White (1999). "Improved rates and asymptotic normality for nonparametric neural network estimators." IEEE Transactions on Information Theory 45(2): 682-691.

Dechert, W. D. and R. Gençay (1992). "Lyapunov exponents as a nonparametric diagnostic for stability analysis." Journal of Applied Econometrics 7: S41-S60.

Ding, Z., C. W. J. Granger and R. F. Engle (1993). "A long memory property of stock market returns and a new model." Journal of Empirical Finance 1, 83-106. 
Eckmann, J. P., S. O. Kamphorst, D. Ruelle and S. Ciliberto (1986). "Liapunov exponents from time series." Physical Review A 34: 4971-4979.

Eckmann, J. P. and D. Ruelle (1985). "Ergodic theory of chaos and strange attractors." Reviews of Modern Physics 57: 617-656.

Ellner, S., A. R. Gallant, D. McCaffrey and D. Nychka (1991). "Convergence rates and data requirements for Jacobian-based estimates of Lyapunov exponents from data." Physics Letter A 153(6,7): 357-363.

Furstenberg, H. and H. Kesten (1960). "Products of random matrices." The Annals of Mathematical Statistics 31: 457-469.

Gabushin, V. N. (1967). "Inequalities for the norms of a function and its derivatives in metric Lp." Matematicheskie Zametki 1(3): 291-298.

Gallant, R. and H. White (1992). "On learning the derivatives of an unknown mapping with multilayer feedforward networks." Neural Networks 5: 129-138.

Gençay, R. (1996). "A statistical framework for testing chaotic dynamics via Lyapunov exponents." Physica D 89: 261-266.

Gençay, R. and W. D. Dechert (1992). "An algorithm for the n Lyapunov exponents of an ndimensional unknown dynamical system." Physica D 59: 142-157.

Grenander, U. (1981). Abstract Inference. New York, Wiley.

Herrndorf, N. (1984). "An invariance principle for weakly dependent sequences of random variables." Annals of Probability 12: 141-153.

Hornik, K., M. Stinchcombe, H. White and P. Auer (1994). "Degree of approximation results for feedforward networks approximating unknown mappings and their derivatives." Neural Computation 6: 1262-1275.

Makovoz, Y. (1996). "Random approximants and neural networks." Journal of Approximation Theory 85: 98-109.

McCaffrey, D. F. (1991). Estimating Lyapunov exponents with nonparametric regression and convergence rates for feedforward single hidden layer networks. Ph.D. Dissertation. Department of Statistics. Raleigh, North Carolina State University. 
McCaffrey, D. F., S. Ellner, A. R. Gallant and D. W. Nychka (1992). "Estimating the Lyapunov exponent of a chaotic system with nonparametric regression." Journal of the American Statistical Association 87(419): 682-695.

Nychka, D., B. Bailey, S. Ellner, P. Haaland and M. O'Connell (1996). "FUNFITS: data analysis and statistical tools for estimating functions" North Carolina Institute of Statistics Mimeoseries No. 2289.

Nychka, D., S. Ellner, D. McCaffrey, and A. R. Gallant (1992). "Finding chaos in noisy system." Journal of the Royal Statistical Society, Series B 54(2): 399-426.

Schwarz, G. (1978). "Estimating the dimension of a model." Annals of Statistics 6(2): 461-464.

Serletis, A. (1995). "Random walks, breaking trend functions, and the chaotic structure of the velocity of money." Journal of Business and Economic Statistics 13(4): 453-458.

Shen, X. (1997). "On methods of sieves and penalization." Annals of Statistics 25(6): 2555-2591.

Shen, X. and W. H. Wong (1994). "Convergence rate of sieve estimates." Annals of Statistics 22(2): 580-615.

Shintani, M. and O. Linton (2001). "Is there chaos in the world economy? A nonparametric test using consistent standard errors." International Economic Review, forthcoming.

Taylor, S. J. (1986) Modelling Financial Time Series, New York, Wiley.

Whang, Y.-J. and O. Linton (1999). "The asymptotic distribution of nonparametric estimates of the Lyapunov exponent for stochastic time series." Journal of Econometrics 91: 1-42. 
Table 1

Logistic Map

\begin{tabular}{|c|c|c|c|c|c|c|}
\hline & \multicolumn{3}{|c|}{ (1) Stable System with $a=1$} & \multicolumn{3}{|c|}{$(\lambda=-0.693)$} \\
\hline & \multicolumn{3}{|c|}{$d=1$} & \multicolumn{3}{|c|}{$d=2$} \\
\hline & Full & Block & Skipped & Full & Block & Skipped \\
\hline mean $(\widehat{\lambda})$ & -0.729 & -0.729 & -0.729 & -0.291 & -0.283 & -0.280 \\
\hline $\operatorname{median}(\widehat{\lambda})$ & -0.710 & -0.705 & -0.707 & -0.276 & -0.271 & -0.271 \\
\hline $\operatorname{std}(\widehat{\lambda})$ & 0.312 & 0.333 & 0.326 & 0.182 & 0.205 & 0.194 \\
\hline mean $(s e)$ & 0.069 & 0.118 & 0.114 & 0.064 & 0.108 & 0.107 \\
\hline median $(s e)$ & 0.068 & 0.114 & 0.112 & 0.062 & 0.105 & 0.104 \\
\hline lower $5 \%$ & 0.060 & 0.060 & 0.060 & 0.050 & 0.060 & 0.060 \\
\hline \multirow[t]{3}{*}{ upper $5 \%$} & 0.040 & 0.040 & 0.040 & 0.040 & 0.030 & 0.040 \\
\hline & \multicolumn{3}{|c|}{$d=3$} & \multicolumn{3}{|c|}{$d=4$} \\
\hline & Full & Block & Skipped & Full & Block & Skipped \\
\hline mean $(\widehat{\lambda})$ & -0.101 & -0.089 & -0.082 & 0.009 & 0.022 & 0.027 \\
\hline $\operatorname{median}(\widehat{\lambda})$ & -0.091 & -0.079 & -0.076 & 0.014 & 0.027 & 0.031 \\
\hline $\operatorname{std}(\widehat{\lambda})$ & 0.124 & 0.147 & 0.140 & 0.094 & 0.116 & 0.111 \\
\hline mean (se) & 0.054 & 0.093 & 0.091 & 0.048 & 0.082 & 0.081 \\
\hline median $(s e)$ & 0.053 & 0.090 & 0.089 & 0.047 & 0.080 & 0.079 \\
\hline lower $5 \%$ & 0.060 & 0.060 & 0.060 & 0.060 & 0.060 & 0.050 \\
\hline upper $5 \%$ & 0.030 & 0.030 & 0.040 & 0.040 & 0.040 & 0.050 \\
\hline \multicolumn{7}{|c|}{ (2) Chaotic System with $a=4 \quad(\lambda=0.693)$} \\
\hline & \multicolumn{3}{|c|}{$d=1$} & \multicolumn{3}{|c|}{$d=2$} \\
\hline & Full & Block & Skipped & Full & Block & Skipped \\
\hline mean $(\widehat{\lambda})$ & 0.689 & 0.689 & 0.689 & 0.664 & 0.667 & 0.669 \\
\hline $\operatorname{median}(\widehat{\lambda})$ & 0.689 & 0.691 & 0.691 & 0.679 & 0.681 & 0.674 \\
\hline $\operatorname{std}(\widehat{\lambda})$ & 0.019 & 0.031 & 0.100 & 0.059 & 0.066 & 0.112 \\
\hline mean $(s e)$ & 0.054 & 0.092 & 0.102 & 0.051 & 0.087 & 0.098 \\
\hline median $(s e)$ & 0.053 & 0.090 & 0.101 & 0.050 & 0.085 & 0.097 \\
\hline lower $5 \%$ & 0.050 & 0.050 & 0.050 & 0.070 & 0.070 & 0.060 \\
\hline \multirow[t]{3}{*}{ upper $5 \%$} & 0.050 & 0.040 & 0.050 & 0.000 & 0.010 & 0.040 \\
\hline & \multicolumn{3}{|c|}{$d=3$} & \multicolumn{3}{|c|}{$d=4$} \\
\hline & Full & Block & Skipped & Full & Block & Skipped \\
\hline mean $(\widehat{\lambda})$ & 0.662 & 0.666 & 0.668 & 0.662 & 0.667 & 0.669 \\
\hline median $(\widehat{\lambda})$ & 0.673 & 0.676 & 0.675 & 0.670 & 0.675 & 0.671 \\
\hline $\operatorname{std}(\widehat{\lambda})$ & 0.054 & 0.061 & 0.112 & 0.046 & 0.054 & 0.107 \\
\hline mean $(s e)$ & 0.050 & 0.086 & 0.098 & 0.050 & 0.086 & 0.097 \\
\hline median $(s e)$ & 0.050 & 0.085 & 0.097 & 0.050 & 0.085 & 0.097 \\
\hline lower $5 \%$ & 0.050 & 0.060 & 0.060 & 0.050 & 0.050 & 0.050 \\
\hline upper $5 \%$ & 0.000 & 0.010 & 0.040 & 0.000 & 0.010 & 0.040 \\
\hline
\end{tabular}

Note: Sample size $(T)=200$. Number of hidden units $(r)=4$. Number of replications $=1000$.

Jacobians are evaluated using full sample (Full) as well as subsamples with blocks (Block) and skips (Skipped) with number of block length $(M)=66$. Lower $5 \%$ and upper $5 \%$ are tail frequencies of normalized Lyapunov exponent estimates using standard normal critical values. 
Table 2

Henon Map

\begin{tabular}{|c|c|c|c|c|c|c|}
\hline \multicolumn{7}{|c|}{ Chaotic System with $a=1.4, b=0.3 \quad(\lambda=0.408)$} \\
\hline & \multicolumn{3}{|c|}{$d=1$} & \multicolumn{3}{|c|}{$d=2$} \\
\hline & Full & Block & Skipped & Full & Block & Skipped \\
\hline mean $(\widehat{\lambda})$ & 0.354 & 0.354 & 0.354 & 0.414 & 0.418 & 0.422 \\
\hline $\operatorname{median}(\widehat{\lambda})$ & 0.358 & 0.361 & 0.362 & 0.417 & 0.422 & 0.425 \\
\hline $\operatorname{std}(\widehat{\lambda})$ & 0.076 & 0.093 & 0.120 & 0.029 & 0.049 & 0.080 \\
\hline mean $(s e)$ & 0.050 & 0.085 & 0.103 & 0.042 & 0.072 & 0.082 \\
\hline median (se) & 0.049 & 0.083 & 0.101 & 0.042 & 0.071 & 0.081 \\
\hline lower $5 \%$ & 0.060 & 0.060 & 0.060 & 0.050 & 0.060 & 0.060 \\
\hline \multirow[t]{3}{*}{ upper $5 \%$} & 0.040 & 0.030 & 0.030 & 0.040 & 0.030 & 0.040 \\
\hline & \multicolumn{3}{|c|}{$d=3$} & \multicolumn{3}{|c|}{$d=4$} \\
\hline & Full & Block & Skipped & Full & Block & Skipped \\
\hline mean $(\widehat{\lambda})$ & 0.411 & 0.417 & 0.424 & 0.411 & 0.416 & 0.420 \\
\hline median $(\widehat{\lambda})$ & 0.411 & 0.420 & 0.429 & 0.411 & 0.420 & 0.424 \\
\hline $\operatorname{std}(\widehat{\lambda})$ & 0.034 & 0.054 & 0.085 & 0.036 & 0.054 & 0.086 \\
\hline mean $(s e)$ & 0.043 & 0.074 & 0.082 & 0.043 & 0.074 & 0.083 \\
\hline median (se) & 0.043 & 0.073 & 0.082 & 0.043 & 0.073 & 0.083 \\
\hline lower $5 \%$ & 0.050 & 0.060 & 0.070 & 0.050 & 0.060 & 0.060 \\
\hline upper $5 \%$ & 0.050 & 0.040 & 0.040 & 0.050 & 0.040 & 0.040 \\
\hline
\end{tabular}

Note: Sample size $(T)=200$. Number of hidden units $(r)=4$. Number of replications $=1000$.

See also note for Table 1. 
Table 3

\section{Barnett Competition Data}

\begin{tabular}{|c|c|c|c|c|c|c|c|}
\hline \multicolumn{4}{|c|}{$T=380$} & \multicolumn{4}{|c|}{$T=2000$} \\
\hline \multirow[b]{2}{*}{$(d, r)$} & \multicolumn{3}{|c|}{ Sample } & \multirow[b]{2}{*}{$(d, r)$} & \multicolumn{3}{|c|}{ Sample } \\
\hline & Full & Block & Skipped & & Full & Block & Skipped \\
\hline \multicolumn{8}{|c|}{ (1) Logistic map } \\
\hline \multirow[t]{3}{*}{$(2,4)$} & 0.015 & 0.294 & 0.126 & $(1,4)$ & 0.012 & 0.010 & 0.016 \\
\hline & $(0.396)$ & $(2.877)$ & $(0.600)$ & & $(1.190)$ & $(0.135)$ & $(0.242)$ \\
\hline & {$[0.654]$} & {$[0.998]$} & {$[0.726]$} & & {$[0.883]$} & {$[0.554]$} & {$[0.596]$} \\
\hline \multicolumn{8}{|c|}{ (2) $G A R C H$} \\
\hline \multirow[t]{3}{*}{$(1,1)$} & -4.260 & -4.357 & -4.323 & $(1,1)$ & -5.017 & -5.072 & -5.009 \\
\hline & $(-56.00)$ & $(-9.171)$ & $(-10.52)$ & & $(-215.1)$ & $(-45.22)$ & $(-46.03)$ \\
\hline & {$[<0.001]$} & {$[<0.001]$} & {$[<0.001]$} & & {$[<0.001]$} & {$[<0.001]$} & {$[<0.001]$} \\
\hline \multicolumn{8}{|c|}{ (3) $N L M A$} \\
\hline \multirow[t]{3}{*}{$(2,3)$} & -0.435 & -0.290 & -0.336 & $(3,4)$ & -0.360 & -0.341 & -0.339 \\
\hline & $(-15.66)$ & $(-1.916)$ & $(-2.031)$ & & $(-43.93)$ & $(-8.656)$ & $(-7.887)$ \\
\hline & {$[<0.001]$} & {$[0.028]$} & {$[0.021]$} & & {$[<0.001]$} & {$[<0.001]$} & {$[<0.001]$} \\
\hline \multicolumn{8}{|c|}{ (4) $A R C H$} \\
\hline \multirow[t]{3}{*}{$(1,1)$} & -3.925 & -4.018 & -3.994 & $(1,1)$ & -3.606 & -3.611 & -3.604 \\
\hline & $(-69.56)$ & $(-12.60)$ & $(-14.49)$ & & $(-1324)$ & $(-291.9)$ & $(-281.6)$ \\
\hline & {$[<0.001]$} & {$[<0.001]$} & {$[<0.001]$} & & {$[<0.001]$} & {$[<0.001]$} & {$[<0.001]$} \\
\hline \multicolumn{8}{|c|}{ (5) $A R M A$} \\
\hline \multirow[t]{3}{*}{$(1,1)$} & -0.049 & -0.029 & -0.051 & $(3,1)$ & -0.041 & -0.029 & -0.034 \\
\hline & $(-4.843)$ & $(-4.937)$ & $(-2.676)$ & & $(-8.116)$ & $(-2.587)$ & $(-3.322)$ \\
\hline & {$[<0.001]$} & {$[<0.001]$} & {$[0.004]$} & & {$[<0.001]$} & {$[0.005]$} & {$[<0.001]$} \\
\hline
\end{tabular}

Note: For the full sample estimation (Full), the largest Lyapunov exponent estimates are presented with $t$ statistics in parentheses and $p$-value for $H_{0}: \lambda \geq 0$ in brackets. For the subsample estimation (Block and Skipped), median values are presented.

The lag length $(d)$ and the number of hidden units $(r)$ are jointly selected based on BIC. QS kernel with optimal bandwidth (Andrews, 1991) is used for the heterosckedasticity and autocorrelation consistent covariance estimation. 
Table 4

Autocorrelations of Stock Return Series

\begin{tabular}{|c|c|c|c|c|c|c|}
\hline data & $\operatorname{lag} 1$ & 2 & 3 & 4 & 5 & 10 \\
\hline (1) return & $\begin{array}{c}0.029 \\
(0.007) \\
\end{array}$ & $\begin{array}{l}-0.022 \\
(0.007) \\
\end{array}$ & $\begin{array}{c}0.005 \\
(0.007) \\
\end{array}$ & $\begin{array}{c}0.018 \\
(0.007) \\
\end{array}$ & $\begin{array}{c}0.019 \\
(0.007) \\
\end{array}$ & $\begin{array}{c}0.007 \\
(0.007) \\
\end{array}$ \\
\hline (2) $\mid$ return $\left.\right|^{0.5}$ & $\begin{array}{c}0.233 \\
(0.007) \\
\end{array}$ & $\begin{array}{c}0.242 \\
(0.007) \\
\end{array}$ & $\begin{array}{c}0.245 \\
(0.008) \\
\end{array}$ & $\begin{array}{c}0.251 \\
(0.008) \\
\end{array}$ & $\begin{array}{c}0.260 \\
(0.008) \\
\end{array}$ & $\begin{array}{c}0.236 \\
(0.010) \\
\end{array}$ \\
\hline (3) $\mid$ return $\left.\right|^{1.0}$ & $\begin{array}{c}0.295 \\
(0.007) \\
\end{array}$ & $\begin{array}{c}0.314 \\
(0.007) \\
\end{array}$ & $\begin{array}{c}0.308 \\
(0.008) \\
\end{array}$ & $\begin{array}{c}0.300 \\
(0.009) \\
\end{array}$ & $\begin{array}{c}0.311 \\
(0.009) \\
\end{array}$ & $\begin{array}{c}0.266 \\
(0.011) \\
\end{array}$ \\
\hline (4) $\mid$ return $\left.\right|^{1.5}$ & $\begin{array}{c}0.280 \\
(0.007) \\
\end{array}$ & $\begin{array}{c}0.294 \\
(0.007) \\
\end{array}$ & $\begin{array}{c}0.269 \\
(0.008) \\
\end{array}$ & $\begin{array}{c}0.243 \\
(0.008) \\
\end{array}$ & $\begin{array}{c}0.271 \\
(0.009) \\
\end{array}$ & $\begin{array}{c}0.198 \\
(0.010) \\
\end{array}$ \\
\hline (5) $\mid$ return $\left.\right|^{2.0}$ & $\begin{array}{c}0.202 \\
(0.007) \\
\end{array}$ & $\begin{array}{c}0.211 \\
(0.007) \\
\end{array}$ & $\begin{array}{c}0.160 \\
(0.007) \\
\end{array}$ & $\begin{array}{c}0.131 \\
(0.008) \\
\end{array}$ & $\begin{array}{c}0.177 \\
(0.008) \\
\end{array}$ & $\begin{array}{c}0.095 \\
(0.008) \\
\end{array}$ \\
\hline (6) $\mid$ return $\left.\right|^{2.5}$ & $\begin{array}{c}0.117 \\
(0.007)\end{array}$ & $\begin{array}{c}0.129 \\
(0.007)\end{array}$ & $\begin{array}{c}0.072 \\
(0.007)\end{array}$ & $\begin{array}{c}0.054 \\
(0.007)\end{array}$ & $\begin{array}{c}0.098 \\
(0.007)\end{array}$ & $\begin{array}{c}0.034 \\
(0.007)\end{array}$ \\
\hline
\end{tabular}

Note: Numbers in parentheses are standard errors. 
Table 5

Lyapunov Exponents of Stock Return Series

\begin{tabular}{|c|c|c|c|c|c|c|}
\hline & \multicolumn{6}{|c|}{ NLAR lag $(d)$} \\
\hline & 1 & 2 & 3 & 4 & 5 & 6 \\
\hline \multicolumn{7}{|c|}{ (1) return } \\
\hline Full & $\begin{array}{c}-2.685 \\
(-262.1) \\
{[<0.001]}\end{array}$ & $\begin{array}{c}-1.539 \\
(-347.7) \\
{[<0.001]}\end{array}$ & $\begin{array}{l}-1.355 \\
(-721.6) \\
{[<0.001]}\end{array}$ & $\begin{array}{c}-0.820 \\
(-228.5) \\
{[<0.001]}\end{array}$ & $\begin{array}{c}-0.562 \\
(-322.7) \\
{[<0.001]}\end{array}$ & $\begin{array}{c}-0.503 \\
(-455.81) \\
{[<0.001]}\end{array}$ \\
\hline Block & $\begin{array}{l}-2.689 \\
(-24.31) \\
{[<0.001]}\end{array}$ & $\begin{array}{l}-1.538 \\
(-30.49) \\
{[<0.001]}\end{array}$ & $\begin{array}{c}-1.339 \\
(-44.93) \\
{[<0.001]}\end{array}$ & $\begin{array}{l}-0.800 \\
(-18.21) \\
{[<0.001]}\end{array}$ & $\begin{array}{c}-0.546 \\
(-13.36) \\
{[<0.001]}\end{array}$ & $\begin{array}{c}-0.487 \\
(-14.70) \\
{[<0.001]}\end{array}$ \\
\hline Skipped & $\begin{array}{c}-2.684 \\
(-23.62) \\
{[<0.001]}\end{array}$ & $\begin{array}{l}-1.540 \\
(-30.35) \\
{[<0.001]}\end{array}$ & $\begin{array}{c}-1.330 \\
(-45.40) \\
{[<0.001]}\end{array}$ & $\begin{array}{c}-0.799 \\
(-17.64) \\
{[<0.001]}\end{array}$ & $\begin{array}{c}-0.541 \\
(-13.40) \\
{[<0.001]}\end{array}$ & $\begin{array}{c}-0.490 \\
(-14.71) \\
{[<0.001]}\end{array}$ \\
\hline $\mathrm{BIC}$ & $-8.944(2)$ & $-8.953(2)$ & $-8.951(3)$ & $-8.953(2)$ & $-8.949(3)$ & $-8.958(3)$ \\
\hline \multicolumn{7}{|c|}{ (2) $\mid$ return $\left.\right|^{0.5}$} \\
\hline Full & $\begin{array}{c}-1.876 \\
(-306.9) \\
{[<0.001]}\end{array}$ & $\begin{array}{c}-0.985 \\
(-189.7) \\
{[<0.001]}\end{array}$ & $\begin{array}{c}-0.568 \\
(-191.3) \\
{[<0.001]}\end{array}$ & $\begin{array}{c}-0.364 \\
(-130.3) \\
{[<0.001]}\end{array}$ & $\begin{array}{c}-0.260 \\
(-113.8) \\
{[<0.001]}\end{array}$ & $\begin{array}{c}-0.194 \\
(-129.7) \\
{[<0.001]}\end{array}$ \\
\hline \multirow[t]{2}{*}{ Block } & $\begin{array}{l}-1.921 \\
(-49.65)\end{array}$ & $\begin{array}{l}-1.017 \\
(-24.24)\end{array}$ & $\begin{array}{l}-0.582 \\
(-20.99)\end{array}$ & $\begin{array}{l}-0.372 \\
(-18.01)\end{array}$ & $\begin{array}{l}-0.264 \\
(-16.27)\end{array}$ & $\begin{array}{l}-0.195 \\
(-1629)\end{array}$ \\
\hline & {$[<0.001]$} & {$[<0.001]$} & {$[<0.001]$} & {$[<0.001]$} & {$[<0.001]$} & {$[<0.001]$} \\
\hline \multirow[t]{3}{*}{ Skipped } & -1.874 & -0.960 & -0.549 & -0.352 & -0.250 & -0.188 \\
\hline & $(-38.53)$ & $(-19.61)$ & $(-19.05)$ & $(-16.51)$ & $(-14.27)$ & $(-14.77)$ \\
\hline & {$[<0.001]$} & {$[<0.001]$} & {$[<0.001]$} & {$[<0.001]$} & {$[<0.001]$} & {$[<0.001]$} \\
\hline $\mathrm{BIC}(r)$ & $-6.459(1)$ & $-6.508(2)$ & $-6.536(3)$ & $-6.554(3)$ & $-6.572(3)$ & $-6.576(3)$ \\
\hline \multicolumn{7}{|c|}{ (3) $\mid$ return $\left.\right|^{1.0}$} \\
\hline Full & $\begin{array}{c}-1.424 \\
(-939.3) \\
{[<0.001]}\end{array}$ & $\begin{array}{c}-0.677 \\
(-233.6) \\
{[<0.001]}\end{array}$ & $\begin{array}{c}-0.476 \\
(-153.1) \\
{[<0.001]}\end{array}$ & $\begin{array}{c}-0.304 \\
(-220.5) \\
{[<0.001]}\end{array}$ & $\begin{array}{c}-0.211 \\
(-177.8) \\
{[<0.001]}\end{array}$ & $\begin{array}{c}-0.173 \\
(-180.2) \\
{[<0.001]}\end{array}$ \\
\hline \multirow[t]{2}{*}{ Block } & $\begin{array}{l}-1.437 \\
(-209.2)\end{array}$ & $\begin{array}{c}-0.693 \\
(-41.18)\end{array}$ & $\begin{array}{c}-0.488 \\
(-25.88)\end{array}$ & $\begin{array}{l}-0.308 \\
(-27.07)\end{array}$ & $\begin{array}{c}-0.213 \\
(-22.45)\end{array}$ & $\begin{array}{c}-0.173 \\
(-19.80)\end{array}$ \\
\hline & {$[<0.001]$} & {$[<0.001]$} & {$[<0.001]$} & {$[<0.001]$} & {$[<0.001]$} & {$[<0.001]$} \\
\hline \multirow[t]{2}{*}{ Skipped } & $\begin{array}{l}-1.424 \\
(-1282)\end{array}$ & $\begin{array}{c}-0.669 \\
(-3619)\end{array}$ & $\begin{array}{l}-0.460 \\
(-2327)\end{array}$ & $\begin{array}{c}-0.298 \\
(-2452)\end{array}$ & $\begin{array}{l}-0.204 \\
(-2108)\end{array}$ & $\begin{array}{l}-0.166 \\
(-20.04)\end{array}$ \\
\hline & {$[<0.001]$} & {$[<0.001]$} & {$[<0.001]$} & {$[<0.001]$} & {$[<0.001]$} & {$[<0.001]$} \\
\hline $\mathrm{BIC}(r)$ & $-9.554(1)$ & $-9.619(2)$ & $-9.660(3)$ & $-9.688(3)$ & $-9.711(3)$ & $-9.716(3)$ \\
\hline
\end{tabular}


Table 5 (Continued)

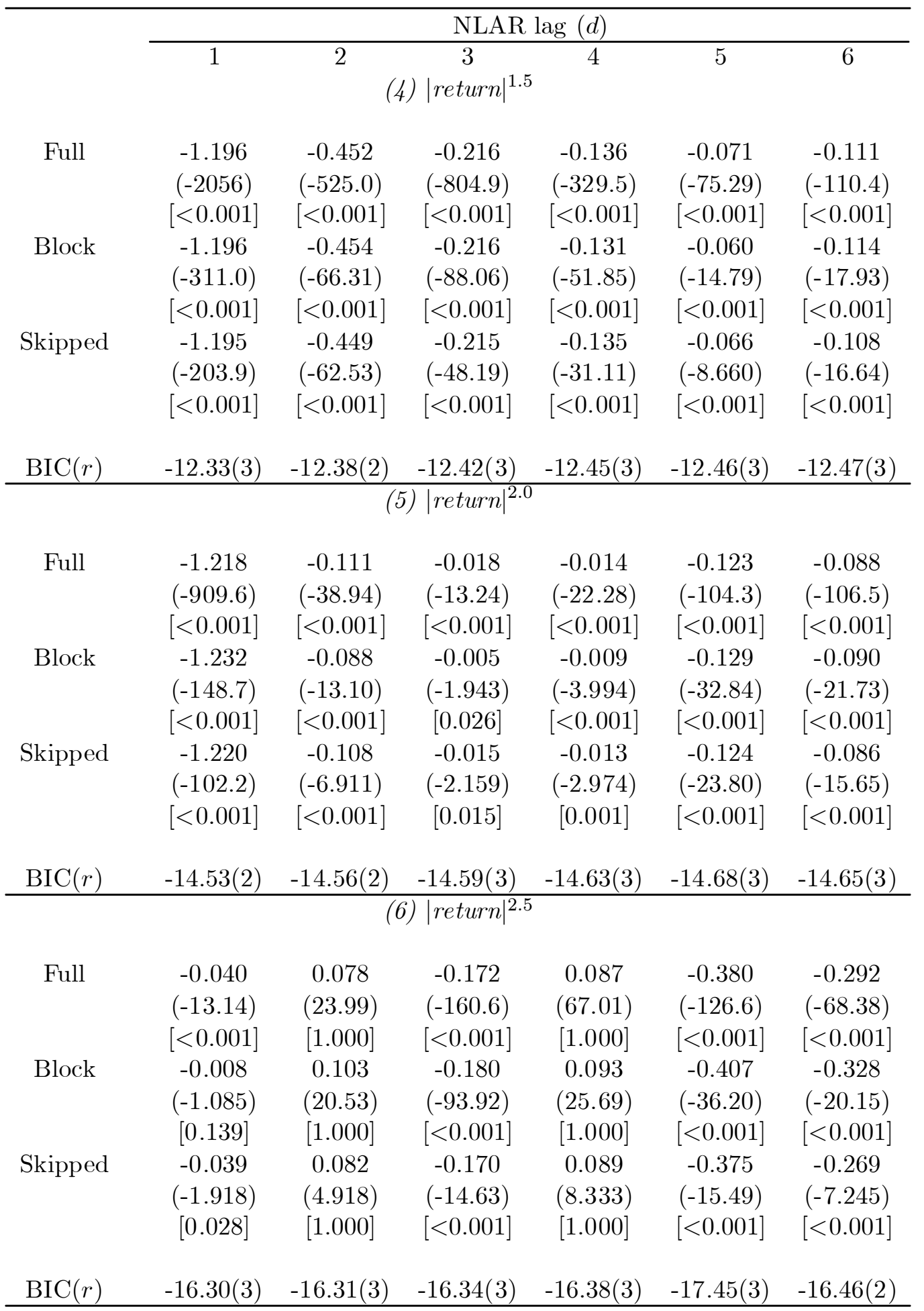

Note: For the full sample estimation (Full), the largest Lyapunov exponent estimates are presented with $t$ statistics in parentheses and $p$-value for $H_{0}: \lambda \geq 0$ in brackets. For the subsample estimation (Block and Skipped), median values are presented. Block length $(M)$ and the number of blocks are 127 and 145, respectively. 
The number of hidden units $(r)$ are selected based on BIC. QS kernel with optimal bandwidth (Andrews, 1991) is used for the heterosckedasticity and autocorrelation consistent covariance estimation. 